\title{
RESEARCH
}

Open Access

\section{Melatonin suppresses ER stress-dependent proapoptotic effects via AMPK in bone mesenchymal stem cells during mitochondrial oxidative damage}

Chongxi Fan ${ }^{1,2^{*}+}$ D, Jianyu Feng ${ }^{3 \dagger}$, Chi Tang ${ }^{1 \dagger}$, Zhengbin Zhang ${ }^{4}$, Yingtong Feng ${ }^{5}$, Weixun Duan ${ }^{3}$, Mingming Zhai ${ }^{1}$, Zedong Yan', Liwen Zhu' ${ }^{6}$, Lele Feng ${ }^{3}$, Hanzhao Zhu ${ }^{3}$ and Erping Luo ${ }^{1 *}$

\begin{abstract}
Background: Bone marrow mesenchymal stem cells (BMSCs) have been used as important cell-based tools for clinical applications. Oxidative stress-induced apoptosis causes a low survival rate after transplantation, and the underlying mechanisms remain unknown. The endoplasmic reticulum (ER) and mitochondria are vital organelles regulated by adenosine monophosphate (AMP)-activated protein kinase (AMPK), especially during oxidative stress injury. Melatonin exerts an antioxidant effect by scavenging free radicals. Here, we aimed to explore whether cytoprotective melatonin relieves ER stress-mediated mitochondrial dysfunction through AMPK in BMSCs after oxidative stress injury.
\end{abstract}

Methods: Mouse BMSCs were isolated and exposed to $\mathrm{H}_{2} \mathrm{O}_{2}$ in the absence or presence of melatonin. Thereafter, cell damage, oxidative stress levels, mitochondrial function, AMPK activity, ER stress-related proteins, and apoptotic markers were measured. Additionally, the involvement of AMPK and ER stress in the melatonin-mediated protection of BMSCs against $\mathrm{H}_{2} \mathrm{O}_{2}$-induced injury was investigated using pharmacologic agonists and inhibitors.

Results: Melatonin improved cell survival and restored mitochondrial function. Moreover, melatonin intimately regulated the phosphorylation of AMPK and molecules associated with ER stress pathways. AMPK activation and ER stress inhibition following melatonin administration improved the mitochondrial membrane potential (MMP), reduced mitochondria-initiated oxidative damage, and ultimately suppressed apoptotic signaling pathways in BMSCs. Cotreatment with N-acetyl-L-cysteine (NAC) significantly enhanced the antioxidant effect of melatonin. Importantly, pharmacological AMPK activation/ER stress inhibition promoted melatonin-induced cytoprotection, while pharmacological AMPK inactivation/ER stress induction conferred resistance to the effect of melatonin against $\mathrm{H}_{2} \mathrm{O}_{2}$ insult.

Conclusions: Our data also reveal a new, potentially therapeutic mechanism by which melatonin protects BMSCs from oxidative stress-mediated mitochondrial apoptosis, possibly by regulating the AMPK-ER stress pathway.

Keywords: Melatonin, Bone marrow mesenchymal stem cells, ER stress, AMPK, Oxidative stress

\footnotetext{
*Correspondence: fcx329@fmmu.edu.com; luoerping@fmmu.edu.cn

${ }^{\dagger}$ Chongxi Fan, Jianyu Feng and Chi Tang contributed equally to this work.

'Department of Military Biomedical Engineering, Air Force Medical University,

169 Changle West Road, Xi'an 710032, China

Full list of author information is available at the end of the article
}

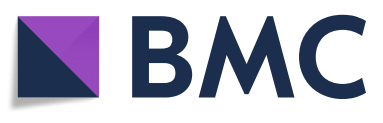

(c) The Author(s). 2020 Open Access This article is licensed under a Creative Commons Attribution 4.0 International License, which permits use, sharing, adaptation, distribution and reproduction in any medium or format, as long as you give appropriate credit to the original author(s) and the source, provide a link to the Creative Commons licence, and indicate if changes were made. The images or other third party material in this article are included in the article's Creative Commons licence, unless indicated otherwise in a credit line to the material. If material is not included in the article's Creative Commons licence and your intended use is not permitted by statutory regulation or exceeds the permitted use, you will need to obtain permission directly from the copyright holder. To view a copy of this licence, visit http://creativecommons.org/licenses/by/4.0/. The Creative Commons Public Domain Dedication waiver (http://creativecommons.org/publicdomain/zero/1.0/) applies to the data made available in this article, unless otherwise stated in a credit line to the data. 


\section{Background}

Bone marrow mesenchymal stem cells (BMSCs) are multipotent stromal cells that have been used as important cell-based tools for various clinical applications due to their high proliferative capacity and their potential to differentiate into multiple lineages, including the bone, cartilage, and fat [1]. Unfortunately, the survival rate of BMSCs in the circulation or injury microenvironment after transplantation is low as a result of hydrodynamic shear stress, excessive inflammatory cytokines, and oxidative stress [2-4].

Although these primary causes of cell death occur together in BMSCs, endoplasmic reticulum (ER) stress has emerged as a potential effector of these events $[5,6]$. The ER, which functions as a site for lipid biosynthesis, protein folding and assembly, and cellular divalent calcium cation $\left(\mathrm{Ca}^{2+}\right)$ storage in eukaryotic cells, is also a major signal-transducing organelle that senses cellular stress under homeostasis [7]. Under stress conditions, the unfolded protein response (UPR) serves as an adaptive response for degrading misfolded proteins that accumulate in the ER, whereas uncontrolled or prolonged ER stress can trigger cell apoptosis; the coupling of these responses in specialized cells or tissues is now thought to be fundamental in oxidative stress injury $[8,9]$. Furthermore, ER stress is initiated by three ER transmembrane proteins: RNA-dependent protein kinase (PKR)-like ER kinase (PERK), inositol-requiring protein 1 (IRE1), and activating transcription factor 6 (ATF6) [10].

Mitochondria are organelles that respond to cell apoptosis regulatory signals by selectively releasing cytochrome $\mathrm{C}$ (Cyto-C) or by increasing reactive oxygen species (ROS) production and decreasing the mitochondrial membrane potential (MMP), which may further worsen ER dysfunction and enhance cell death [11]. However, the upstream mediator of mitochondrial and ER damage has not been identified. Mitochondria are also considered to be producers of energy through adenosine triphosphate (ATP) synthesis, which plays a critical role in the regulation of energy metabolism through adenosine monophosphate (AMP)-activated protein kinase (AMPK) signaling [12, 13].

AMPK is an evolutionarily conserved energy sensor associated with an elegant energy metabolism system; it has diverse cytoprotective effects on glucose and lipid metabolism, the cell cycle, and protein synthesis and participates in cell apoptosis $[14,15]$. It is believed that increased AMP levels, ATP deprivation, and oxidative stress can activate AMPK [16]. A growing body of evidence suggests that AMPK specifically regulates various aspects of mitochondrial biology and homeostasis, including mitochondrial numbers, through stimulation of mitochondrial biogenesis and control of the shape of the mitochondrial network and the quality of mitochondria in cells [17]. In addition, although $\mathrm{Lu}$ and colleagues showed that elevated circulating saturated fatty acid concentrations induce ER stress-mediated apoptosis, which is blocked by AMPK activation [18], in human BMSCs, whether AMPK is a pivotal modulator of ER stress during mitochondrial oxidative damage remains unaddressed. In addition, identifying an effective and rapid therapeutic approach to attenuate the influence of the circulatory or injury microenvironment on BMSCs, especially one that involves endogenous active ingredients, remains an urgent clinical need.

Melatonin ( $\mathrm{N}$-acetyl-5-methoxytryptamine, Mel) is an indoleamine-containing hormone that is secreted mainly from the pineal gland in mammals, and its existence in other tissues, including the ovary, testis, gut, placenta liver, and especially the bone marrow, where it is found at high levels, has also been verified [19]. Many studies have demonstrated that melatonin regulates a variety of important physiological functions, such as circadian rhythms and reproductive and neuroendocrine activities [20]. In recent years, increasing evidence has focused on the antioxidant and anti-inflammatory effects of melatonin on BMSCs after injury. For example, Lee et al. demonstrated that treatment with melatonin suppresses the activation of ER stress-associated proteins and the occurrence of apoptotic cell death in human adipose-derived mesenchymal stem cells (MSCs), which enhances the therapeutic efficacy of MSCs in a murine hindlimb ischemia model [21]. Furthermore, melatonin maintains human MSC survival and promotes osteogenic differentiation in the IL-1ß-induced inflammatory environment, suggesting that melatonin treatment could be a promising method for bone regenerative engineering [22]. Importantly, our findings indicate that melatonin contributes to the amelioration of high-flow shear stress-induced BMSC injury by activating melatonin receptors and AMPK/acetyl-Co A carboxylase (ACC) signaling [4]. Therefore, this study, as an extension of our previous research, focuses on the ability of cytoprotective melatonin to relieve ER stress-mediated mitochondrial dysfunction through AMPK in mouse BMSCs after hydrogen peroxide $\left(\mathrm{H}_{2} \mathrm{O}_{2}\right)$-induced oxidative stress injury.

\section{Methods}

\section{Reagents and antibodies}

Melatonin (>98\% purity, M5250), 4-phenylbutyric acid (4-PBA, > 99\% purity, SML0309), and dimethyl sulfoxide (DMSO, D8418) were purchased from Sigma-Aldrich (St. Louis, MO, USA); thapsigargin (TG, > 98\% purity, ab120286) was from Abcam (Cambridge, MA, USA); compound $\mathrm{C}$ dihydrochloride $(\mathrm{CpC}, \mathrm{T} 6146)$ and acadesine (AICAR, T1477) were from TargetMol (Wellesley Hills, MA, USA); $N$-acetyl-L-cysteine (NAC, S1623) was from Selleck Chemicals (Houston, TX, USA); and 5,5',6, 
6'-tetraethyl-benzimidazolylcarbocyanine iodide (JC-1, C2006), the bicinchoninic acid (BCA, P0011) protein assay kit, the crystal violet staining solution (C0121), the BeyoClick $^{\text {Tw }}$ EdU cell proliferation kit with Alexa Fluor 594 (C0078S), 4',6-diamidino-2-phenylindole (DAPI, C1002), the commercial assay kit for lactate dehydrogenase (LDH, C0016) analysis, the cell mitochondria isolation kit (C3601), immune staining wash buffer (Triton $\mathrm{X}-100, \mathrm{P} 0106)$, radioimmunoprecipitation assay (RIPA) lysis buffer (P0013C), and SDS-PAGE sample loading buffer (5x, P0015) were from Beyotime Biotechnology (Shanghai, China). MitoSOX ${ }^{\mathrm{im}}$ Red mitochondrial superoxide indicator (M36008) was from Molecular Probes Inc. (Invitrogen, Eugene, OR, USA). The small interfering RNA (siRNA) oligonucleotides against AMPK (sc29674) and DDIT3 (sc-35438), the nonspecific control siRNA oligonucleotide (sc-37007), and the transfection reagent (sc-29528) were purchased from Santa Cruz Biotechnology (Dallas, Texas, 75220, USA), and $30 \% \mathrm{H}_{2} \mathrm{O}_{2}$ was purchased from Tianjin Beilian Fine Chemical Development Co., Ltd. (Tianjin, China). C57BL/6 mouse MSC basal medium (BM, MUBMX-90011), osteogenic differentiation basal medium (MUBMX-90021), and adipogenic differentiation basal medium (MUBMX-90031) were from Cyagen Biosciences (Soochow, China); penicillin-streptomycin solution (15140122) was purchased from Life Technologies Co., Ltd. (Carlsbad, CA, USA), and TRIzol reagent was from Invitrogen (Carlsbad, CA, USA). HiScript II Q RT SuperMix (R223-01) and ChamQ SYBR qPCR Master Mix (Q311-02) were purchased from Vazyme Biotech Co., Ltd. (Nanjing, China), and $0.25 \%$ trypsin-ethylenediaminetetraacetic acid (EDTA) solution (T1300), Hanks balanced salt solution (HBSS) with calcium and magnesium (H1025), and bovine serum albumin (BSA, A8010) were purchased from Solarbio Science \& Technology Co., Ltd. (Beijing, China). The Cell Counting Kit-8 (CCK-8, C008-3) assay kit was obtained from 7Sea Pharmatech Co., Ltd. (Shanghai, China). The terminal deoxynucleotidyl transferase dUTP nick end labeling (TUNEL, 11684795910) staining kit for apoptosis detection was from Roche Molecular Biochemicals (Mannheim, Germany), and the Annexin V-FITC/propidium iodide (PI) staining kit (40302ES50) and reactive oxygen species (50101ES01) assay kit were acquired from Yeasen Biotech Co., Ltd. (Shanghai, China). The flow cytometry staining buffer (00-4222-26) was from eBioscience (San Diego, CA, USA). The antibodies and primers used in this study are listed in Tables 1 and 2. Other chemicals and reagents were of analytical grade.

\section{Ethics statement}

All animal experimental procedures performed in the present study were conducted in accordance with the
Guide for the Care and Use of Laboratory Animals published by the US National Institutes of Health (National Institutes of Health Publication No. 85-23, revised in 1996), and approval was obtained from the Ethics Committee of the Fourth Military Medical University (No. 20180301).

\section{Animals}

Adult male C57BL/6 mice (8-week old, 20-25 g) were obtained from the animal center of Air Force Medical University (Xi'an, China). The mice were kept in an Association for Assessment and Accreditation of Laboratory Animal Care (AAALAC)-accredited facility in an environmentally controlled room (12-h light-dark cycle, $60 \%$ humidity) at $20-23^{\circ} \mathrm{C}$, and they were allowed ad libitum access to standard chow and sterile water.

\section{Isolation and culture of mouse BMSCs}

The mouse BMSCs were flushed from the tibial and femoral marrow compartments under sterile conditions with 3\% fetal bovine serum (FBS, Cyagen Biosciences, Soochow, China) in PBS, and the procedure adhered strictly to standard protocols [23]. After passage through a $70-\mu \mathrm{m}$ cell strainer (Corning, NY, USA), all the cells were cultured with C57BL/6 mouse BM with $10 \%$ FBS in a standard $\mathrm{CO}_{2}$ incubator ( $95 \%$ air, $5 \% \mathrm{CO}_{2}$ ). MSCs were isolated through frequent and gentle medium changes within 2 weeks, and adherent marrow cells were subcultured to passage 4 (P4). Cells at less than passage 10 were used for subsequent trials, according to previous recommendations [23].

\section{Flow cytometric BMSC characterization}

To identify the cell surface markers of BMSCs, P4 cells were collected according to the previous literature, detached from plates with $0.25 \%$ trypsin-EDTA solution, and gently resuspended in flow cytometry staining solution at a density of $5 \times 10^{6}$ cells $/ \mathrm{mL}[3,24]$. Then, the following antibodies were applied for $30 \mathrm{~min}$ at room temperature protected from light: anti-CD29-PE, antiCD34-PE, anti-CD44-PE, anti-CD45-PE, anti-CD73-PE, and anti-CD105-PE. The nonspecific Fc receptor was blocked with anti-CD16/32 antibodies. Nonspecific fluorescence was determined by incubation with isotypematched anti-mouse monoclonal antibodies. After staining, the samples were processed with EPICS XL-MCL (Beckman Coulter, Brea, CA, USA), and the analysis was performed with EXPO32 ADC.

\section{Adipogenesis and osteogenesis in mouse BMSCs}

Adipogenic and osteogenic differentiation of BMSCs was performed strictly according to the commercial product manual from Cyagen Biosciences (Soochow, China). Briefly, BMSCs (P6) were seeded in six-well plates 
Table 1 List of antibodies

\begin{tabular}{|c|c|c|c|c|c|}
\hline Target proteins & Cat. no. & Source & Mol.Wt. & Provider & Application \\
\hline CD16/32 & $14-0161$ & Rat & N/A & eBioscience (San Diego, CA, USA) & FACS \\
\hline Phycoerythrin (PE) anti-mouse/rat CD29 Antibody & 102207 & $\begin{array}{l}\text { Armenian } \\
\text { Hamster }\end{array}$ & N/A & BioLegend (San Diego, CA, USA) & FACS \\
\hline PE anti-mouse CD34 Antibody & 128609 & $\begin{array}{l}\text { Armenian } \\
\text { Hamster }\end{array}$ & N/A & BioLegend (San Diego, CA, USA) & FACS \\
\hline PE anti-mouse/human CD44 Antibody & 103007 & Rabbit & N/A & BioLegend (San Diego, CA, USA) & FACS \\
\hline PE anti-mouse CD45 Antibody & 103105 & Rabbit & N/A & BioLegend (San Diego, CA, USA) & FACS \\
\hline PE anti-mouse CD73 Antibody & 127205 & Rabbit & N/A & BioLegend (San Diego, CA, USA) & FACS \\
\hline PE anti-mouse CD105 Antibody & 120407 & Rabbit & N/A & BioLegend (San Diego, CA, USA) & FACS \\
\hline PE Armenian Hamster lgG Isotype Ctrl Antibody & 400907 & $\begin{array}{l}\text { Armenian } \\
\text { Hamster }\end{array}$ & N/A & BioLegend (San Diego, CA, USA) & FACS \\
\hline PE Rat IgG2a, k Isotype Ctrl Antibody & 400507 & Rabbit & N/A & BioLegend (San Diego, CA, USA) & FACS \\
\hline PE Rat IgG2b, K Isotype Ctrl Antibody & 400607 & Rabbit & N/A & BioLegend (San Diego, CA, USA) & FACS \\
\hline APAF-1 & 21710-1-AP & Rabbit & 142 kDa & ProteinTech (Wuhan, China) & WB \\
\hline phospho-PERK (p-PERK) & DF7576 & Rabbit & $125 \mathrm{kDa}$ & $\begin{array}{l}\text { Affinity Biosciences (Cincinnati, OH, } \\
\text { USA) }\end{array}$ & WB \\
\hline PERK & 20582-1-AP & Rabbit & $125 \mathrm{kDa}$ & ProteinTech (Wuhan, China) & WB \\
\hline $\mathrm{BiP}$ & ab21685 & Rabbit & 78 kDa & Abcam (Cambridge, MA, USA) & WB \& IF \\
\hline Calreticulin (CRT) & ab22683 & Mouse & N/A & Abcam (Cambridge, MA, USA) & IF \\
\hline phospho-AMPK (p-AMPK) & \#2535 & Rabbit & $62 \mathrm{kDa}$ & $\begin{array}{l}\text { Cell Signaling Technology (Beverly, } \\
\text { MA, USA) }\end{array}$ & WB \\
\hline AMPK & \#2532 & Rabbit & $62 \mathrm{kDa}$ & $\begin{array}{l}\text { Cell Signaling Technology (Beverly, } \\
\text { MA, USA) }\end{array}$ & WB \\
\hline phospho-elF2a (p-elF2a) & \#3398 & Rabbit & $38 \mathrm{kDa}$ & $\begin{array}{l}\text { Cell Signaling Technology (Beverly, } \\
\text { MA, USA) }\end{array}$ & WB \\
\hline elF2a & \#5324 & Rabbit & $38 \mathrm{kDa}$ & $\begin{array}{l}\text { Cell Signaling Technology (Beverly, } \\
\text { MA, USA) }\end{array}$ & WB \\
\hline Caspase-3 (Casp-3) & \#9665 & Rabbit & $35,19,17 \mathrm{kDa}$ & $\begin{array}{l}\text { Cell Signaling Technology (Beverly, } \\
\text { MA, USA) }\end{array}$ & WB \\
\hline Cleaved-Caspase-3 (C-Casp-3) & \#9664 & Rabbit & $19,17 \mathrm{kDa}$ & $\begin{array}{l}\text { Cell Signaling Technology (Beverly, } \\
\text { MA, USA) }\end{array}$ & WB \& IF \\
\hline DNA damage-inducible transcript 3 (DDIT3) & ab11419 & Mouse & $31 \mathrm{kDa}$ & Abcam (Cambridge, MA, USA) & WB \\
\hline $\mathrm{BCl} 2$ & 12789-1-AP & Rabbit & $26 \mathrm{kDa}$ & ProteinTech (Wuhan, China) & WB \\
\hline MnSOD & ab13533 & Rabbit & $25 \mathrm{kDa}$ & Abcam (Cambridge, MA, USA) & WB \\
\hline Bax & \#2772 & Rabbit & $20 \mathrm{kDa}$ & $\begin{array}{l}\text { Cell Signaling Technology (Beverly, } \\
\text { MA, USA) }\end{array}$ & WB \\
\hline Cytochrome C oxidase IV (COX IV) & 11242-1-AP & Rabbit & $18-17 \mathrm{kDa}$ & ProteinTech (Wuhan, China) & WB \\
\hline Cytochrome C (Cyto-C) & ab13575 & Mouse & $12 \mathrm{kDa}$ & Abcam (Cambridge, MA, USA) & WB \\
\hline$\beta$-Tubulin & 10094-1-AP & Rabbit & $55 \mathrm{kDa}$ & ProteinTech (Wuhan, China) & WB \\
\hline$\beta$-actin & SC-47778 & Mouse & $43 \mathrm{kDa}$ & Santa Cruz (Dallas, TX, USA) & WB \\
\hline GAPDH & $60004-1-\lg$ & Mouse & $36 \mathrm{kDa}$ & ProteinTech (Wuhan, China) & WB \\
\hline HRP-conjugated goat anti-mouse antibody & ZB-2305 & Goat & N/A & Zhongshan Company (Beijing, China) & WB \\
\hline HRP-conjugated goat anti-rabbit antibody & ZB-2301 & Goat & N/A & Zhongshan Company (Beijing, China) & WB \\
\hline Alexa Fluor 488-conjugated goat anti-mouse IgG & EK011 & Goat & N/A & Zhuangzhibio (Xi'an, China) & IF \\
\hline Cy3-conjugated goat anti-rabbit lgG & EK022 & Goat & N/A & Zhuangzhibio (Xi'an, China) & IF \\
\hline
\end{tabular}

(precoated with $5 \%$ gelatin) at a density of $2 \times 10^{4}$ cells $/ \mathrm{cm}^{2}$ at $37{ }^{\circ} \mathrm{C}$ with $5 \% \mathrm{CO}_{2}$ for at least $12 \mathrm{~h}$. For osteogenic differentiation, $2 \mathrm{~mL}$ of preheated complete inducing medium was replaced every 3 days when the percentage of cell fusion reached $60-70 \%$. After 2-4 weeks of induction, depending on the morphological changes and growth of the 
cells, the cells were stained with Alizarin Red. For adipogenesis, BMSCs were incubated with adipogenic differentiation medium A until they reached $100 \%$ confluence or fusion. After 2-3 days, the supernatant was replaced with adipogenic differentiation medium $B$ for 1 day. During 3-5 alternating cycles (12-20 days), medium B was used for 47 days until the lipid droplets became sufficiently large and round. Adipogenesis was determined by staining with Oil Red O solution to visualize lipids in the cytoplasm.

\section{Experimental conditions}

Step one: The cytotoxic effects of $\mathrm{H}_{2} \mathrm{O}_{2}$-induced oxidative stress on mouse BMSCs and the regulation of AMPK and ER stress during this process were first evaluated. The cells were incubated in normal culture medium in 96-well plates until they reached $80 \%$ confluence. The medium was then replaced with different concentrations of $\mathrm{H}_{2} \mathrm{O}_{2}(0-800 \mu \mathrm{M}$, diluted in serum-free $\mathrm{BM}$ ) for another $24 \mathrm{~h}$ to explore suitable conditions for subsequent experimental modeling. Step two: The cytoprotective effects of melatonin were confirmed. The maximum safe dose of melatonin for BMSCs has been studied, and this dose was used in experiments investigating the ability of melatonin to protect against $\mathrm{H}_{2} \mathrm{O}_{2}-$ induced injury. Step three: Some small-molecule modulators (such as Met, $\mathrm{Cpc}$, TG, and 4-PBA) and siRNA agents were used at the indicated doses to modulate their target proteins to further investigate the roles of AMPK and ER stress in the protective effect of melatonin against oxidative stress damage in BMSCs. Unless otherwise stated, all the compounds were dissolved in DMSO or PBS. The solution was stored at $4{ }^{\circ} \mathrm{C}$ for 7 days, diluted in BM (without FBS), and filtered immediately before the experiment to achieve a DMSO concentration of less than $0.1 \%$.

\section{Cell viability analysis}

Mouse BMSCs in logarithmic phase were inoculated in 96-well plates (precoated with $5 \%$ gelatin) at a density of $3 \times 10^{4}$ cells $/ \mathrm{cm}^{2}$ at $37^{\circ} \mathrm{C}$ with $5 \% \mathrm{CO}_{2}$ for at least $12 \mathrm{~h}$ to achieve a stable state. Then, different treatments were implemented at the indicated doses for the indicated times. Ultimately, $100 \mu \mathrm{L}$ of BM with $10 \mu \mathrm{L}$ of CCK-8 solution was added and incubated at $37{ }^{\circ} \mathrm{C}$ with $5 \% \mathrm{CO}_{2}$ for $2 \mathrm{~h}$ in the dark. The optical density (OD) values were analyzed at $450 \mathrm{~nm}$ using a microplate reader (SpectraMax 190, Molecular Device, USA) within $3 \mathrm{~min}$.

\section{Cell proliferation assay}

Cell proliferation was assessed with an EdU-Alexa Fluor 594 staining kit according to the manufacturer's instructions. Briefly, microscope slides with BMSCs were prepared and incubated with $10 \mu \mathrm{M} E d U$ for $2 \mathrm{~h}$ at $37^{\circ} \mathrm{C}$ in the dark. Then, the samples were fixed with $4 \%$ paraformaldehyde at room temperature for $15 \mathrm{~min}$, followed by $0.3 \%$ Triton X-100 for another $10 \mathrm{~min}$. Finally, click additive solution was used to excite fluorescence (excitation/emission $=590 / 615 \mathrm{~nm}$ ), and the nuclei were visualized with Hoechst 33342 (excitation/emission $=346 / 460$ $\mathrm{nm})$. The fluorescence signals in at least five random separate fields were acquired using an FV1000 Olympus confocal microscope (Olympus, Tokyo, Japan). The fluorescence intensity was analyzed with ImageJ software (1.52a, National Institutes of Health, Bethesda, MD, USA).

\section{LDH cytotoxicity assay}

Oxidative stress-induced cell death was assessed spectrophotometrically via an LDH release assay of cell culture supernatants according to the manufacturer's instructions. The absorbance of all samples was read at $490 \mathrm{~nm}$ using a SpectraMax M5 microplate reader (SpectraMax M5, Molecular Device, USA) within 3 min.

\section{Determination of BMSC apoptosis}

The cell apoptosis rate was detected using the TUNEL and Annexin V-FITC/PI staining kits after different treatments. For TUNEL analysis, BMSCs were grown on gelatin-precoated microscopy slides and fixed with $4 \%$ paraformaldehyde at room temperature for $15 \mathrm{~min}$. The samples were then covered with $100 \mu \mathrm{L}$ of mixed TUNEL reagent for $1 \mathrm{~h}$ at $37^{\circ} \mathrm{C}$ in a thermostatic water bath. DAPI solution $(5 \mu \mathrm{g} / \mathrm{mL})$ was then added to reveal the number of nuclei. The TUNEL signals were observed with an FV1000 Olympus confocal microscope (Olympus, Tokyo, Japan). At least 100 cells/fields (5 fields in general) were randomly counted to determine the ratio of the number of TUNEL-positive nuclei to the number of DAPI-positive nuclei and calculate apoptosis rates.

For Annexin V-FITC/PI staining, BMSCs were seeded in six-well plates at $6 \times 10^{5}$ cells $/ \mathrm{mL}$ for overnight stabilization. After administration, the cells were gently collected with trypsin solution. The suspension was centrifuged at $300 \mathrm{~g}$ and $4{ }^{\circ} \mathrm{C}$ for $5 \mathrm{~min}$. In total, $1 \times 10^{5}$ cells were resuspended in $195 \mu \mathrm{L}$ of FITC-conjugated annexin $\mathrm{V}$ binding buffer containing $5 \mu \mathrm{L}$ of Annexin V-FITC and $10 \mu \mathrm{L}$ of PI at room temperature for $20 \mathrm{~min}$ in the

Table $\mathbf{2}$ List of primers

\begin{tabular}{llll}
\hline Target genes & Forward sequence & Reverse sequence & Provider \\
\hline Apaf-1 & AGTAATGGGTCCTAAGCATGTTG & GCGATTGGGAAAATCACGTAAAA & Rescript (Nanjing, China) \\
GAPDH & AGGTCGGTGTGAACGGATTTG & TGTAGACCATGTAGTTGAGGTCA & Rescript (Nanjing, China) \\
\hline
\end{tabular}


dark and maintained on ice when processing. The samples were analyzed with EPICS XL-MCL (Beckman Coulter, Brea, CA, USA), and analysis was performed with EXPO32 ADC within $30 \mathrm{~min}$.

\section{Determination of intracellular free radical production}

The ROS in BMSCs were measured with DCFH-DA staining on gelatin-precoated microscopy slides without fixation at $37^{\circ} \mathrm{C}$ for $30 \mathrm{~min}$ in the dark. The fluorescence signals in at least five random separate fields were acquired using an FV1000 Olympus confocal microscope (Olympus, Tokyo, Japan) at a wavelength of $488 / 525 \mathrm{~nm}$ (excitation/emission). The fluorescence intensity was analyzed with ImageJ software (1.52a, National Institutes of Health, Bethesda, MD, USA).

\section{Mitochondrial superoxide analysis}

The MitoSOX ${ }^{\text {su }}$ Red indicator is a novel fluorochrome for the highly selective detection of superoxide in the mitochondria of live cells on glass coverslips after different treatments. The cells were incubated with $5 \mu \mathrm{M}$ MitoSOX $^{\mathrm{mm}}$ reagent working solution (stored in DMSO at $5 \mathrm{mM}$ and diluted with HBSS before use) for $10 \mathrm{~min}$ at $37^{\circ} \mathrm{C}$, and the samples were protected from light. The fluorescence signal was imaged with an FV1000 Olympus confocal microscope (Olympus, Tokyo, Japan) at an excitation $/$ emission $=510 / 580 \mathrm{~nm}$. The fluorescence intensity was analyzed with ImageJ software (1.52a, National Institutes of Health, Bethesda, MD, USA).

\section{MMP detection}

The levels of MMP in BMSCs were analyzed by staining with the cationic dye JC-1 according to our previous study [25]. JC-1 aggregates in the mitochondrial matrix predominantly produce red fluorescence, as assessed using excitation/emission wavelengths of $525 / 590 \mathrm{~nm}$; otherwise, after mitochondrial polarization, JC-1 is in the monomer form, which can produce green fluorescence at $490 / 530 \mathrm{~nm}$. The specimens were incubated with JC-1 solution for $20 \mathrm{~min}$ at $37^{\circ} \mathrm{C}$ in the dark. The MMP was represented by the ratio of red fluorescence intensity to green fluorescence intensity.

\section{Transwell chemotaxis assays}

BMSCs were cultured in Transwell chambers with $8-\mu \mathrm{m}$ pores (3422; Corning Inc., ME, USA). The upper chamber was seeded with MSCs $\left(2 \times 10^{4}\right.$ cells), which were grown in $100 \mu \mathrm{L}$ of $\mathrm{BM}$. Additionally, the lower chamber contained $600 \mu \mathrm{L}$ of $\mathrm{BM}$ with various concentrations of melatonin [24]. After $24 \mathrm{~h}$, the cells remaining on the upper surface of the filter were removed with cotton swabs, and those that traversed to the lower surface were revealed by crystal violet staining solution for $30 \mathrm{~min}$ at room temperature. The numbers of migrated cells were counted in five randomly selected fields using a 600D camera (Canon Company, Tokyo, Japan).

\section{Scratch migration assays}

BMSCs were plated on six-well plates and grown until they reached close to $90 \%$ confluence. After washing with PBS, a scratch was created in the cell layer along the diameter of the well with a $200-\mu \mathrm{L}$ pipet tip. Next, the cells were incubated with $2 \mathrm{~mL}$ of serum-free $\mathrm{BM}$ containing various concentrations of melatonin. Three photomicrographs of each scratch were obtained at the initial time $(0$ h) of wound creation, and the same location was photographed $24 \mathrm{~h}$ later to determine the extent of wound closure [26]. ImageJ software (1.52a, National Institutes of Health, Bethesda, MD, USA) was used to measure the relative width of the scratch in each group.

\section{Immunofluorescence microscopy}

After treatment with the indicated agents for the indicated times, the BMSCs on coverslips were washed with PBS and fixed in 4\% paraformaldehyde at room temperature for $15 \mathrm{~min}$. Then, 3\% BSA in PBS containing $0.3 \%$ Triton $\mathrm{X}-100$ solution was used to increase the membrane permeability and prevent nonspecific adsorption of immune agents at $37^{\circ} \mathrm{C}$ for $1 \mathrm{~h}$. The primary antibodies were incubated overnight at $4{ }^{\circ} \mathrm{C}$. On the following day, Alexa Fluor 488-conjugated goat anti-mouse IgG antibodies against calreticulin (CRT) and Cy3conjugated goat anti-rabbit IgG antibodies against Bip were used to label the cells at $37^{\circ} \mathrm{C}$ for $1 \mathrm{~h}$. DAPI solution $(5 \mu \mathrm{g} / \mathrm{mL})$ was added to stain the cell nuclei. The fluorescence signal was captured with an FV1000 Olympus confocal microscope (Olympus, Tokyo, Japan) at excitation/emission $=493 / 519 \mathrm{~nm}$ (Alexa Fluor 488) and excitation $/$ emission $=550 / 570 \mathrm{~nm} \quad(\mathrm{Cy} 3)$. The fluorescence intensity was analyzed with Image software (1.52a, National Institutes of Health, Bethesda, MD, USA). The primary antibodies used in the present study are shown in Table 1.

\section{Cytochrome C (Cyto-C) release assay}

The mitochondrial and cytosolic fractions were separated according to the manufacturer's instructions. Briefly, BMSCs were plated in six-well plates and treated with the indicated agents. Cells were collected with $0.25 \%$ trypsin-EDTA solution, washed with ice-cold PBS, and resuspended in homogenization buffer containing 1 mM PMSF for $15 \mathrm{~min}$. The cells were homogenized approximately 10-30 times using a Teflon/glass tissue grinder. The effect of the homogenate was evaluated with trypan blue. The cell lysate was centrifuged at $600 \mathrm{~g}$ for $10 \mathrm{~min}$ to remove unbroken cells and nuclei, and the supernatant was centrifuged at $11,000 \mathrm{~g}$ for $10 \mathrm{~min}$ at $4{ }^{\circ} \mathrm{C}$ to obtain the supernatant (without touching the 
precipitate) and the pure pellet. The supernatant was used as the cytosolic fraction, whereas the pellet containing the mitochondria was washed and resuspended in homogenization buffer. Protein concentrations were estimated with the BCA protein assay kit. The translocation of Cyto-C from mitochondria to the cytoplasm was further detected by western blotting.

\section{Knockdown of gene expression with siRNA}

BMSCs were cultured in six-well plates in the presence of $10 \%$ FBS without antibiotics for $6 \mathrm{~h}$. The working solution (containing targeted-siRNA oligonucleotides and transfection reagent) was prepared at room temperature for $30 \mathrm{~min}$. The cells were then cultured in the above solution with culture medium for $48 \mathrm{~h}$ to allow maximal inhibition of protein expression. Scrambled small interfering RNA (Consi) was used as the control. The knockdown efficiency was further assessed by western blotting.

\section{Western blot analysis}

The protein lysates from BMSCs were prepared after different treatments. Equal amounts of protein (approximately $20-35 \mu \mathrm{g}$ ) were loaded onto either 10 or $12 \%$ SDS-polyacrylamide gels. The separated proteins were transferred onto PVDF membranes using a wet protein transfer system (Bio-Rad, West Berkeley, CA, USA) for $1.5 \mathrm{~h}$ in an icebox. After blocking with $5 \%$ nonfat dry milk, the membranes were incubated overnight at $4{ }^{\circ} \mathrm{C}$ with the primary antibodies listed in Table 1 . Subsequently, the membranes were incubated with specific HRP-conjugated secondary antibodies at $37^{\circ} \mathrm{C}$ for $1 \mathrm{~h}$. Ultimately, the immunoreactive signals were visualized using an electrochemiluminescence system (Bio-Rad, West Berkeley, CA, USA), and the relative intensities of the bands were quantified using Image Lab software (Bio-Rad, West Berkeley, CA, USA). According to the experiment, $\beta$-actin, $\beta$-tubulin, GAPDH, and COX IV were selected as internal controls.

\section{RNA extraction and real-time quantitative PCR (RT-qPCR) assay}

Total RNA isolation and real-time PCR were conducted as described in our previous study [27]. Briefly, BMSCs were treated as indicated. Total RNA was isolated from the cells with TRIzol reagent. After isolation, the RNA quality was assessed on a NanoDrop2000 spectrophotometer (Thermo Scientific, Waltham, MA, USA) according to the manufacturer's instructions. Absorbance ratios (260/280) ranging from 1.8-2.0 indicated pure RNA samples. Reverse transcription was conducted with oligo-dT to prime the reverse transcription reaction, and the apoptotic protease activating factor-1 (Apaf-1) primers used to amplify cDNA are listed in Table 2 . Real-time PCR cycling was carried out using a Bio-Rad
CFX96 Real-Time System (Bio-Rad, West Berkeley, CA, USA). The PCR mixture consisted of $10 \mu \mathrm{L}$ of $2 \times \mathrm{TB}$ Green Premix Ex Taq II, $0.8 \mu \mathrm{L}$ of both sense and antisense primers, $2.0 \mu \mathrm{L}$ of sample cDNA solution, and distilled water to a final volume of $20 \mu \mathrm{L}$. The cycling program was as follows: $95^{\circ} \mathrm{C}$ for $30 \mathrm{~s}$ and 40 cycles of $95^{\circ} \mathrm{C}$ for $5 \mathrm{~s}$ and $60^{\circ} \mathrm{C}$ for $30 \mathrm{~s}$, with a melting curve from 65 to $95^{\circ} \mathrm{C}$ to ensure amplification of a single product. The mRNA levels were calculated using the $2^{-\triangle \Delta C t}$ method (relative fold change). GAPDH was amplified as an internal control.

\section{Statistical analysis}

The results are expressed as the mean \pm standard deviation (SD) unless otherwise specified. Skewness and kurtosis were used to describe the data distribution. Then, the Shapiro-Wilk test was further applied to evaluate normality. Group comparisons were performed using oneway ANOVA followed by the LSD test (SPSS 13.0, SPSS Inc., Chicago, IL, USA). All in vitro and imaging studies were performed in a blinded manner. $p<0.05$ was considered to indicate a statistically significant difference.

\section{Results}

\section{Characterization of mouse BMSCs}

When BMSCs were cultured, the cells began to attach 6-8 $\mathrm{h}$ after the tibial and femoral marrow compartments were flushed; this process was used to separate the BMSCs from contaminating hematopoietic cells. Morphologically, the adherent cells initially showed a polygonal appearance and then grew rapidly and became spindle- or fibroblast-shaped (Fig. 1a). As shown by flow cytometry analysis, most adherent cells highly expressed CD29, CD44, CD73, and CD105 (MSC markers) and were nearly negative for CD34 and CD45 (hematopoietic antigens) (Fig. 1b). These results indicated that BMSCs separated from the bone marrow of mouse long bones were of high purity. The multipotent differentiation capacity of the BMSCs was also analyzed. After 3 weeks of adipogenic induction, the cells were fixed with $4 \%$ paraformaldehyde at room temperature for $30 \mathrm{~min}$ and incubated with Oil Red O solution. Many oil droplets were observed in the cytoplasm. After approximately 4 weeks of osteogenic induction, mineralized nodules were visualized by Alizarin Red staining (Fig. 1c). These morphological changes indicated that the cells had complete multipotent differentiation capacity.

\section{Effect of $\mathrm{H}_{2} \mathrm{O}_{2}$ on BMSC apoptosis}

To construct a model of oxidative stress, we treated BMSCs with different concentrations of $\mathrm{H}_{2} \mathrm{O}_{2}$ ranging from 200 to $800 \mu \mathrm{M}$ for $24 \mathrm{~h}$ in 96 -well plates. The CCK- 8 assay was performed, and the OD values were analyzed at $450 \mathrm{~nm}$. The results showed that the viability 


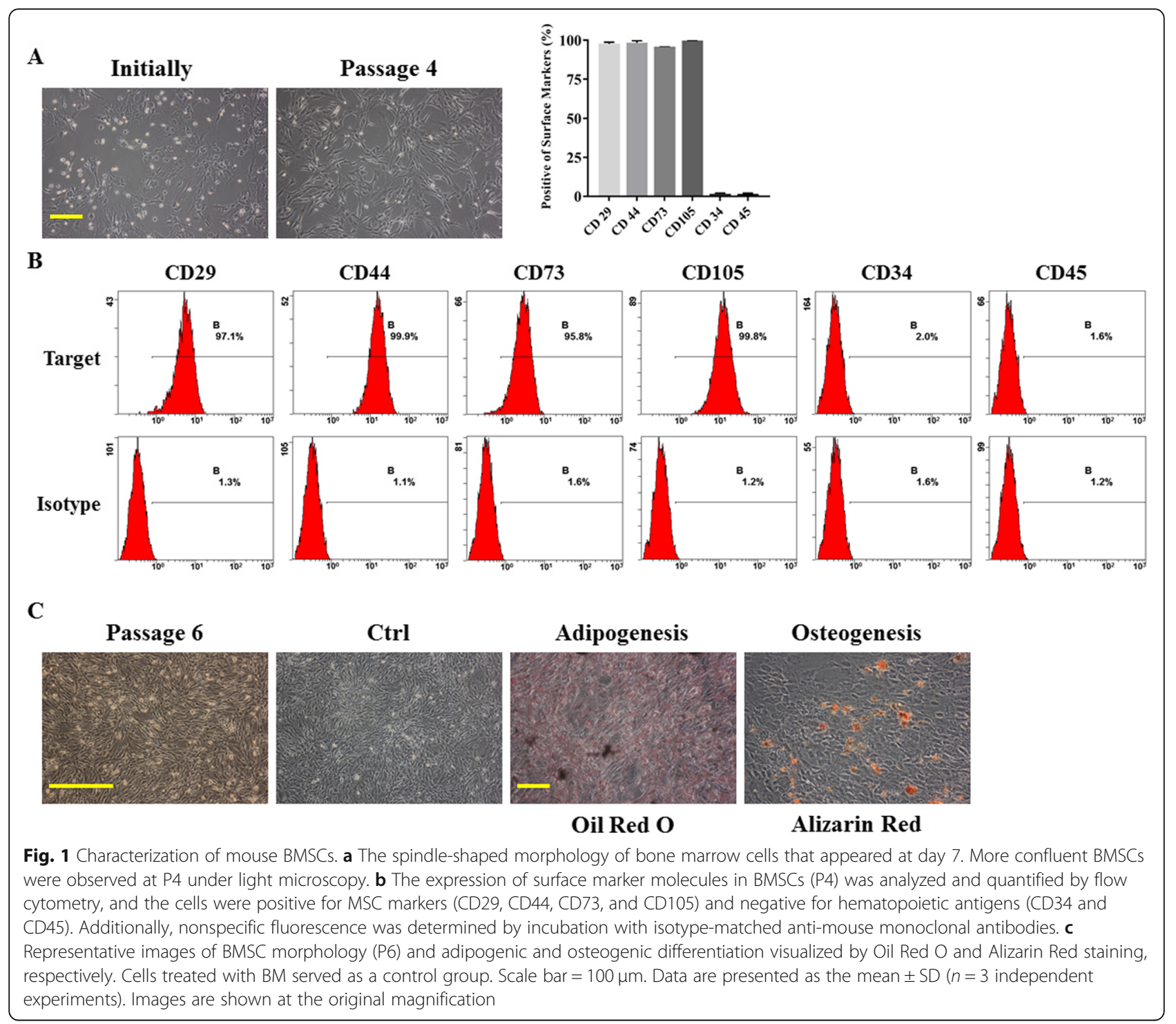

of BMSCs decreased with increasing concentrations of $\mathrm{H}_{2} \mathrm{O}_{2}$, and the IC50 value was approximately $400 \mu \mathrm{M}$ (Fig. 2a). LDH, which is retained in the cytoplasm of viable cells with intact plasma membranes and released from cells after membrane damage, is considered an important indicator of necrosis [28]. Spectrophotometric analysis demonstrated that the LDH level was significantly increased in the medium of cells treated with $\mathrm{H}_{2} \mathrm{O}_{2}$, especially those treated with $800 \mu \mathrm{M} \mathrm{H} \mathrm{H}_{2} \mathrm{O}_{2}$ (Fig. 2b). Additionally, TUNEL staining, which is used to detect apoptosis, revealed that $\mathrm{H}_{2} \mathrm{O}_{2}$ induced a significant increase in green fluorescence (Fig. 2c); this was also verified by Annexin V and PI labeling of the cells (Fig. 2d). These results indicated that $\mathrm{H}_{2} \mathrm{O}_{2}$ obviously increased the cell apoptotic index in a dose-dependent manner. Moreover, with increasing $\mathrm{H}_{2} \mathrm{O}_{2}$ concentrations, the cells showed irreversible damage, which was manifested by the development of early to late apoptosis (Fig. 2d). The cells were subsequently harvested for western blot analysis. As expected, increasing $\mathrm{H}_{2} \mathrm{O}_{2}$ concentrations were associated with BMSC apoptosis, as evidenced by a decrease in the expression of the antiapoptotic protein Bcl2 and increases in the expression of the proapoptotic protein Bax and the apoptosis effector cleaved Caspase-3 (C-Casp-3) (Fig. 2e).

\section{Effects of $\mathrm{H}_{2} \mathrm{O}_{2}$ on mitochondrial dysfunction, ER stress, and AMPK signaling in BMSCs}

ROS are important mediators of $\mathrm{H}_{2} \mathrm{O}_{2}$-induced cell death and are mainly produced in mitochondria [11, 29]. After being treated with different concentrations of $\mathrm{H}_{2} \mathrm{O}_{2}$, BMSCs were stained with DCFH-DA solution. DCFH-DA can be hydrolyzed to DCFH, which is further oxidized to fluorescent DCF by ROS in the cytoplasm. 


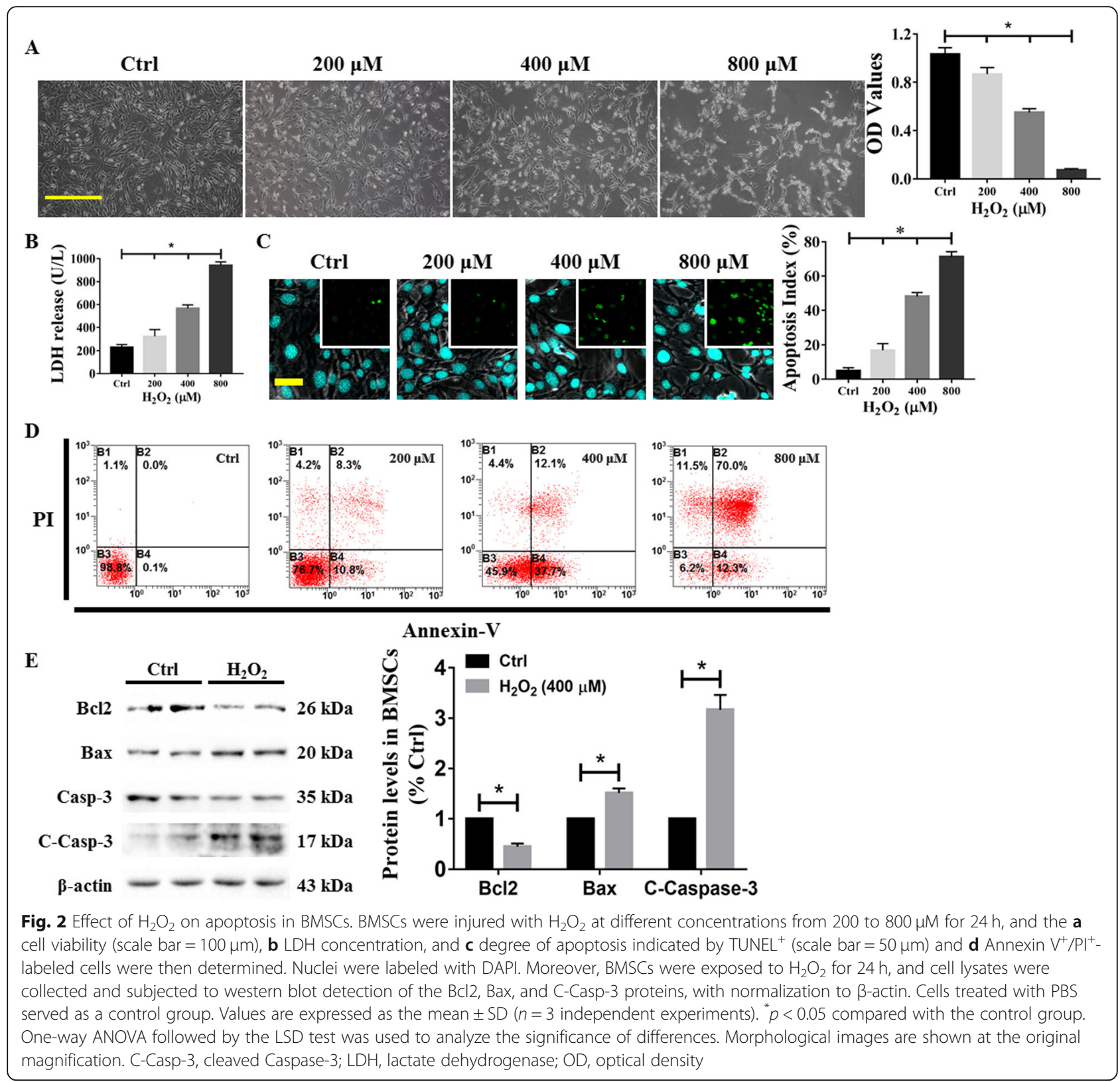

As shown in Fig. $3 \mathrm{a}, \mathrm{H}_{2} \mathrm{O}_{2}$ stimulation resulted in enhanced green fluorescence in BMSCs, which indicated an increase in ROS production. Additionally, the Mito$\mathrm{SOX}^{\mathrm{Tm}}$ Red mitochondrial superoxide indicator, a novel fluorogenic dye that is used for the highly selective detection of superoxides in the mitochondria of live cells and that is readily oxidized by superoxides but not by other ROS- or reactive nitrogen species (RNS)-generating systems, was used. Our results showed that $\mathrm{H}_{2} \mathrm{O}_{2}$-induced red fluorescence rapidly increased in BMSCs, and this finding was consistent with the trend of ROS being present throughout the cell (Fig. 3b).

Mitochondrial bioenergetics is closely associated with a normal MMP level, which is important for ROS scavenging and cytoprotection against apoptosis induced by excessive ROS [30, 31]. The fluorescent lipophilic cation JC-1 is an MMP-sensitive dye that aggregates in the mitochondrial matrix. It exhibits red fluorescence in healthy cells and emits green fluorescence as a monomer [31]. As shown in Fig. 3c, the MMP, as assessed by staining with $\mathrm{JC}-1$, decreased in a dose-dependent manner in response to $\mathrm{H}_{2} \mathrm{O}_{2}$ treatment. Moreover, the MMP is closely related to the integrity of the mitochondrial membrane [32]. When the mitochondrial outer membrane is disrupted, intermembrane space proteins, notably Cyto-C, are released into the cytosol [32]. Accordingly, western blotting was performed, and it demonstrated that Cyto-C translocated from the 


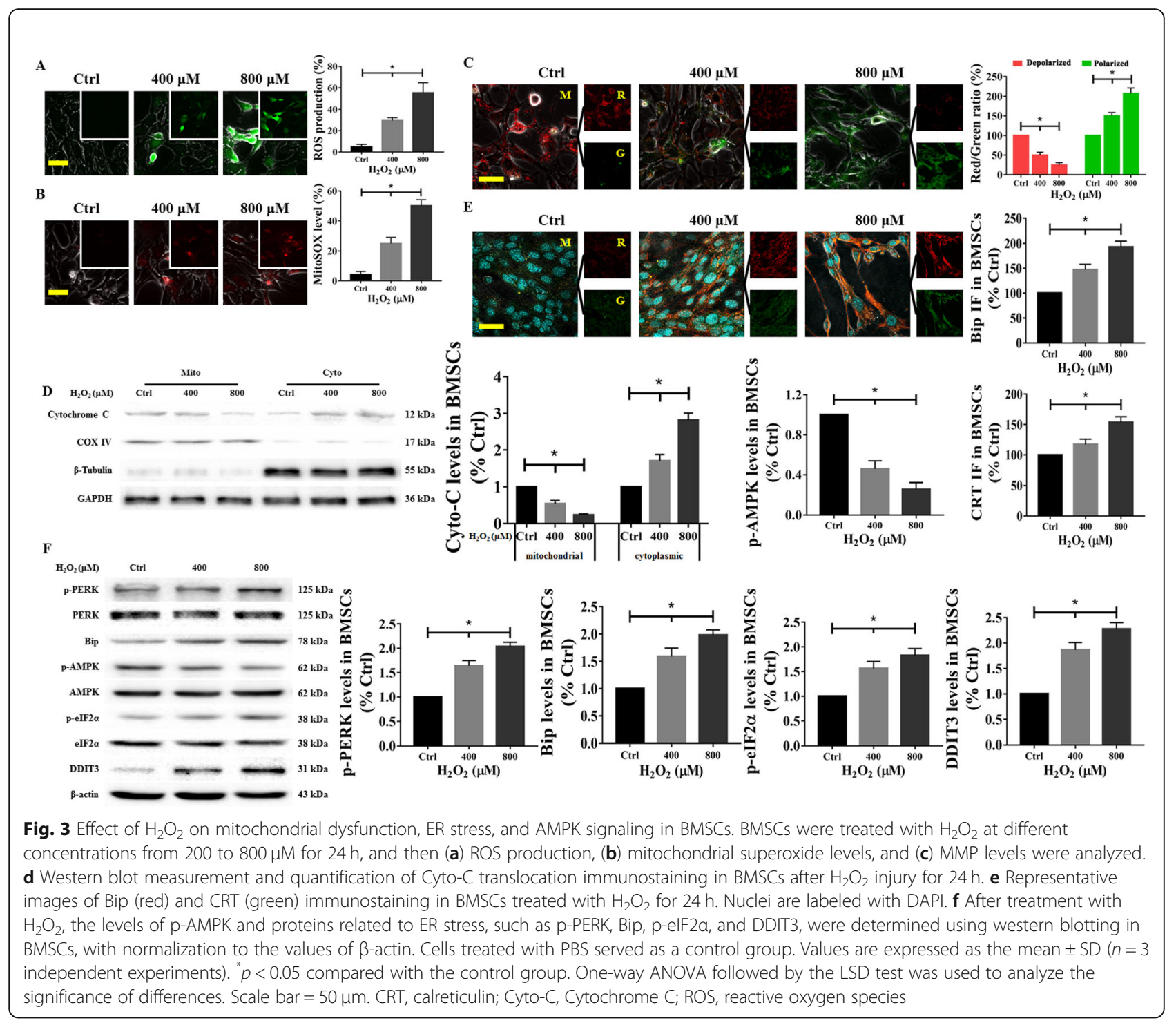

mitochondria to the cytoplasm; COX VI and $\beta$-tubulin served as loading controls, indicating successful mitochondrial isolation (Fig. 3d).

ROS have emerged as crucial regulators of ER function and AMPK activation. Therefore, we investigated the effects of $\mathrm{H}_{2} \mathrm{O}_{2}$ on these two pivotal processes in BMSCs. Immunofluorescence revealed increases in red (indicating Bip expression) and green (indicating expression of CRT, a marker of the ER lumen [33]) fluorescence intensities (Fig. 3e). Additionally, when the BMSCs were harvested, the levels of proteins related to ER stress, such as phospho (p)-PERK, Bip, p-eIF2 $\alpha$, and DDIT3, were rapidly increased in the $\mathrm{H}_{2} \mathrm{O}_{2}$-treated group. Simultaneously, the levels of p-AMPK were significantly decreased by $\mathrm{H}_{2} \mathrm{O}_{2}$ treatment (Fig. 3f). This evidence suggests that ROS produced in mitochondria directly or indirectly affect ER homeostasis and AMPK activity, as reported in previous studies [11-13].

\section{Effect of melatonin on the cell biology of BMSCs}

To determine whether melatonin (the molecular structure of which is shown in Fig. 4a) could directly or indirectly regulate cell biological characteristics, we examined the cytoactivity and migration of BMSCs. First, the cytotoxicity of different concentrations of melatonin was analyzed. BMSCs seeded in 96-well plates were treated with melatonin (ranging from 50 to $800 \mu \mathrm{M}$ ) for $24 \mathrm{~h}$, and cell viability was detected by the CCK- 8 assay. The results showed that the administration of melatonin at a concentration lower than $100 \mu \mathrm{M}$ had almost no effect, while the OD values decreased by $10 \%$ upon treatment with $200 \mu \mathrm{M}$ melatonin (Fig. 4b). Therefore, the maximal 

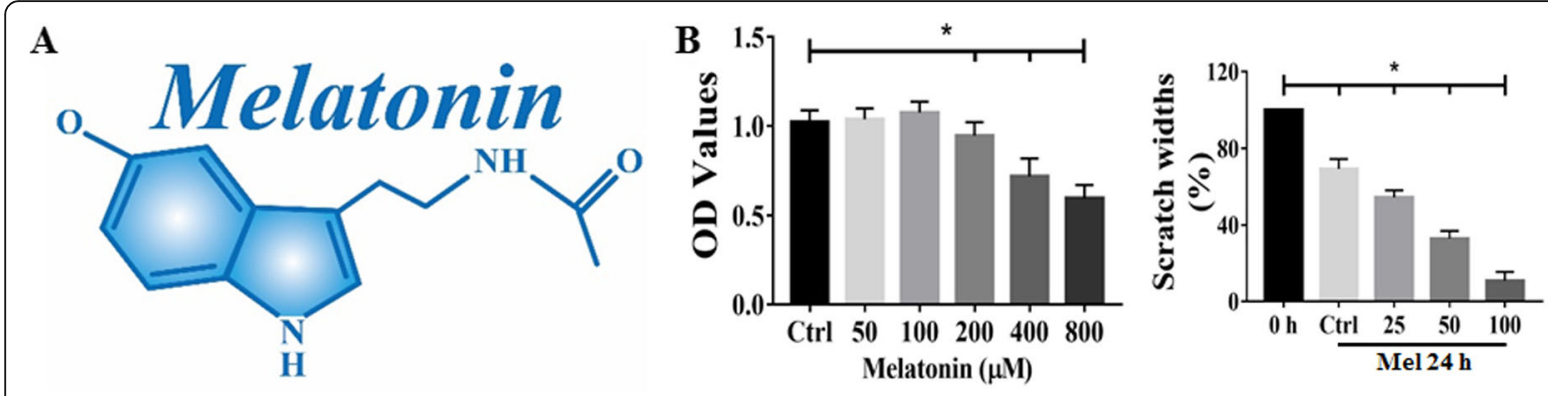

C
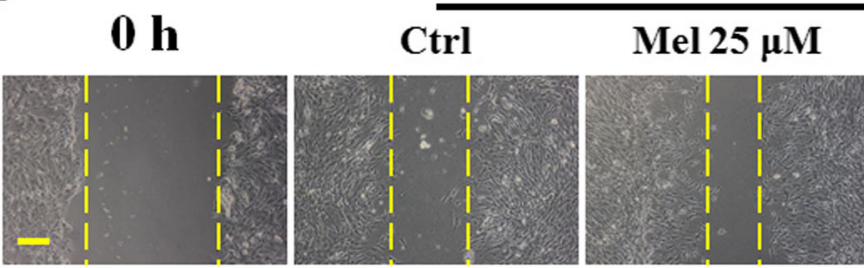

24 h

D

$24 \mathrm{~h}$

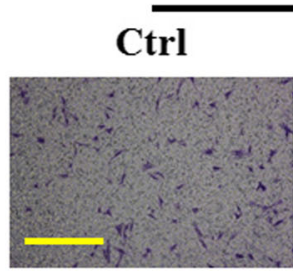

Mel $25 \mu \mathrm{M}$

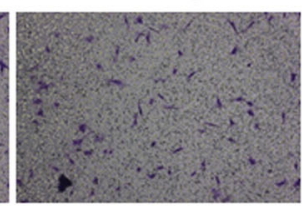

Mel $100 \mu \mathrm{M}$

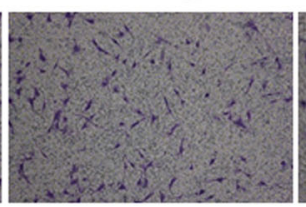

Mel $50 \mu \mathrm{M}$

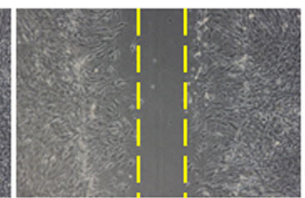

\section{Mel $100 \mu \mathrm{M}$}

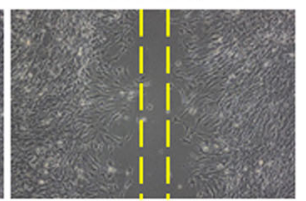

Fig. 4 Effect of melatonin on cell biological behaviors in BMSCs. BMSCs were incubated with different concentrations of melatonin for $24 \mathrm{~h}$. BMSCs seeded in 96-well plates were treated with melatonin (ranging from 50 to $800 \mu \mathrm{M}$ ) for $24 \mathrm{~h}$. a Chemical structure of melatonin (Mel). b Cell viability was determined by a CCK-8 assay. Scratch migration (c) and Transwell chemotaxis assays (d) were performed for $24 \mathrm{~h}$ with melatonin treatment. Scale bar $=100 \mu \mathrm{m}$. Cells treated with PBS served as a control group. Values are expressed as the mean \pm SD $(n=3$ independent experiments). ${ }^{*} p<0.05$ compared with the control group. One-way ANOVA followed by the LSD test was used to analyze the significance of differences. Images are shown at the original magnification. Mel, melatonin; OD, optical density

concentration of melatonin used in our current study was $100 \mu \mathrm{M}$. In the next step, the effect of melatonin on BMSC migration was evaluated by assessing Transwell chemotaxis and performing a wound-healing assay. As evidenced by the wound-healing assay, treatment with various concentrations of melatonin decreased the distance between BMSCs on the two sides of a scratch created by a $200-\mu \mathrm{L}$ pipet tip after $24 \mathrm{~h}$ (Fig. 4c). Moreover, as illustrated in Fig. 4d, the two-chamber migration assay and crystal violet staining showed that melatonin treatment for $24 \mathrm{~h}$ increased the number of cells on the bottom of the membrane insert. These data suggested that melatonin could promote BMSC migration and that melatonin could boost the biological behaviors of BMSCs to a certain extent.

\section{Effect of melatonin on cell damage induced by $\mathrm{H}_{2} \mathrm{O}_{2}$ in BMSCs}

To investigate the protective effect of melatonin, BMSCs pretreated with different concentrations of melatonin (50 and $100 \mu \mathrm{M}$ ) for $6 \mathrm{~h}$ were exposed to $400 \mu \mathrm{M} \mathrm{H}_{2} \mathrm{O}_{2}$ for another $24 \mathrm{~h}$, and their viability was analyzed by the CCK-8 assay. The cell viability of the $\mathrm{H}_{2} \mathrm{O}_{2}$ group was significantly decreased compared with that of the control group, and a gradual increase in the melatonin concentration promoted cell survival, as evidenced by a more typical cell morphology and higher OD values (Fig. 5a). Additionally, $\mathrm{H}_{2} \mathrm{O}_{2}$ insult increased the apoptotic index, and melatonin treatment markedly reduced $\mathrm{H}_{2} \mathrm{O}_{2}$-induced apoptosis (Fig. 5b). The Annexin V-FITC/PI staining results revealed that $\mathrm{H}_{2} \mathrm{O}_{2}$ injury caused a significant increase in the incidence of apoptosis, while melatonin administration decreased the apoptosis rate (Fig. 5c). In addition, BMSCs were labeled with EdUAlexa Fluor 594 to determine whether melatonin could enhance cell proliferation. The immunostaining results revealed an increase in the number of EdU-594 $4^{+}$BMSCs after treatment with melatonin compared with the $\mathrm{H}_{2} \mathrm{O}_{2}$ treatment (Fig. 5d). Consistent with the above results, melatonin not only increased $\mathrm{Bcl} 2$ expression but also decreased Bax and C-Casp-3 expression after oxidative stress injury, providing molecular evidence that the 


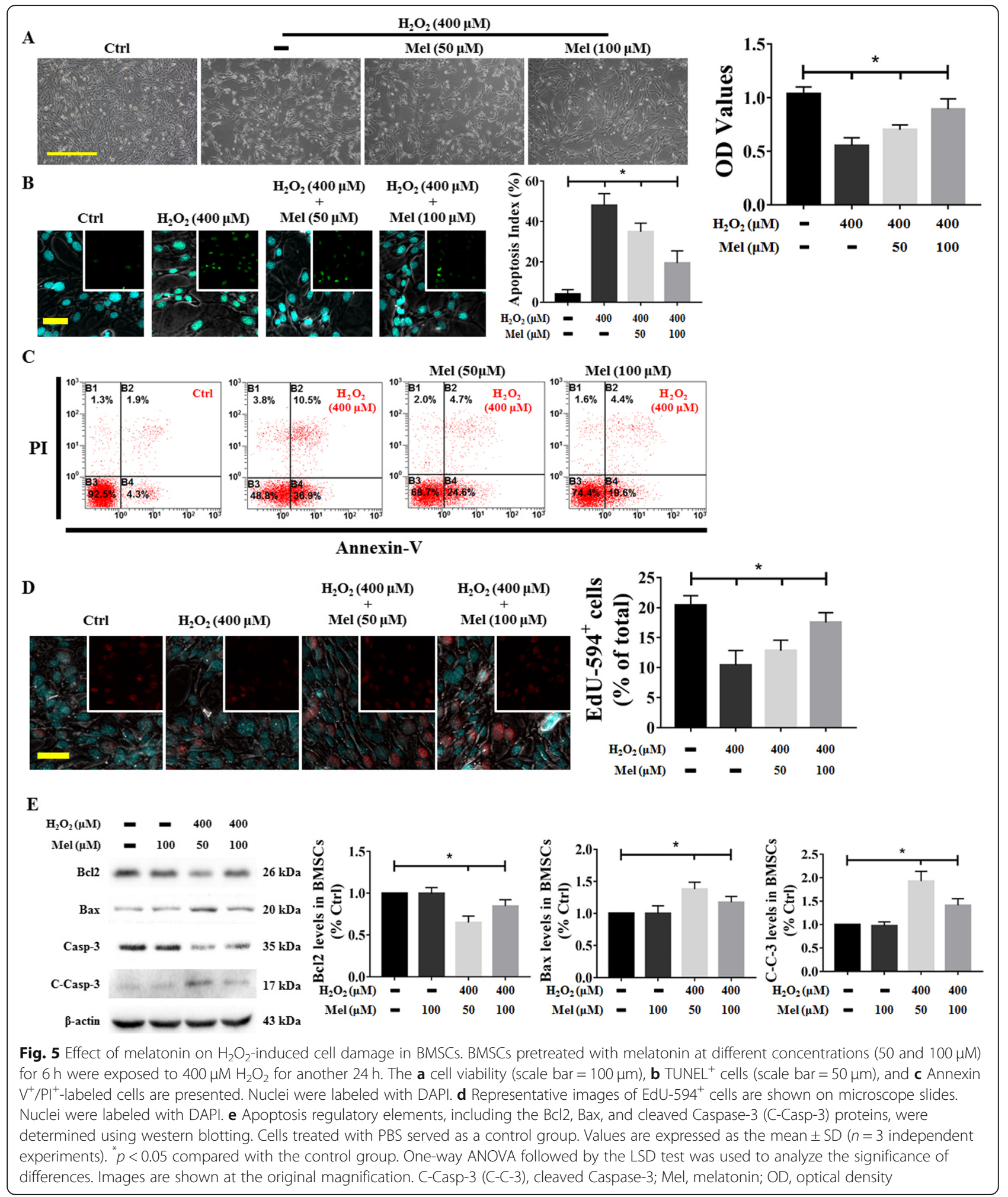

protective effect of melatonin against $\mathrm{H}_{2} \mathrm{O}_{2}$ damage might be due to inhibition of apoptosis (Fig. 5e).

Moreover, NAC (a free radical scavenger that acts as the precursor of glutathione and as a scavenger of free radicals [34]) was used to explore whether the mitochondria-dependent protective effects of melatonin on cell apoptosis were due to the inhibition of ROS production. As indicated in Supplementary Figure 1, BMSCs 
were preprocessed with NAC $(1 \mathrm{mM})$ in the absence or presence of melatonin $(100 \mu \mathrm{M})$ for $6 \mathrm{~h}$ and then exposed to $400 \mu \mathrm{M} \mathrm{H}_{2} \mathrm{O}_{2}$ for another $24 \mathrm{~h}$. Cotreatment with NAC and melatonin further promoted cell viability (Supplementary Figure 1A) and inhibited ROS production (Supplementary Figure 1B). Moreover, the apoptotic index and C-Casp-3 fluorescence were significantly decreased in the $\mathrm{NAC}+$ melatonin group compared with the melatonin group (Supplementary Figure $1 \mathrm{C}-1 \mathrm{E}$ ). The RT-qPCR results showed that BMSCs treated with $100 \mu \mathrm{M} \mathrm{H} \mathrm{H}_{2} \mathrm{O}_{2}$ had significantly higher expression of Apaf-1 than did the control group, while the expression of this factor was reduced after melatonin treatment, with reduced expression in the NAC + melatonin group (Supplementary Figure 1F). Additionally, NAC and melatonin treatment obviously increased the protein expression of $\mathrm{Bcl} 2$ and decreased the levels of $\mathrm{Bax}, \mathrm{C}$ Casp-3, and Apaf-1 during $\mathrm{H}_{2} \mathrm{O}_{2}$-induced injury (Supplementary Figure 1G).

\section{Effect of melatonin on mitochondrial function, ER stress, and AMPK signaling in response to $\mathrm{H}_{2} \mathrm{O}_{2}$-induced BMSC insult}

As mentioned above, mitochondrial dysfunction, ER stress, and AMPK inactivation are three important regulatory mechanisms for oxidative stress injury. Theoretically, restoring mitochondrial function, inhibiting ER stress, or increasing AMPK activity would have a beneficial effect on the ability of BMSCs to withstand $\mathrm{H}_{2} \mathrm{O}_{2}$ induced damage. Considering the ability of melatonin to scavenge oxygen-free radicals, we next explored the potential mechanism by which it protects BMSCs from $\mathrm{H}_{2} \mathrm{O}_{2}$-induced apoptosis. As shown in Fig. $6 \mathrm{a}-\mathrm{c}, \mathrm{H}_{2} \mathrm{O}_{2}$ accelerated whole-cell and mitochondrial superoxide production and depolarization of the mitochondrial membrane, while pretreatment with melatonin significantly attenuated the accumulation of ROS and increased the MMP. In addition, melatonin reduced the expression of Bip and CRT after $\mathrm{H}_{2} \mathrm{O}_{2}$ insult (Fig. 6d), as indicated by weakened fluorescence intensities (red is indicated as Bip and green is indicated as CRT). The western blot analysis also demonstrated that treatment with melatonin prevented the translocation of Cyto- $\mathrm{C}$ to the cytosol and increased the levels of ER stress factors ( $p$ PERK, Bip, p-eIF2 $\alpha$, and DDIT3) (Fig. 6e and f). Furthermore, AMPK activity was also enhanced by melatonin in $\mathrm{H}_{2} \mathrm{O}_{2}$-treated BMSCs, as evidenced by the elevation of p-AMPK (Fig. 6f).

\section{Regulatory effects of activated AMPK and ER stress on melatonin-mediated cellular protection against $\mathrm{H}_{2} \mathrm{O}_{2}$ damage}

To investigate the roles of AMPK and ER stress in melatonin-induced cellular protection against $\mathrm{H}_{2} \mathrm{O}_{2}$ exposure, we further upregulated the expression of AMPK and induced ER stress with AICAR and TG, respectively. The cells were processed as follows: (1) For AMPK $\alpha$ activation, BMSCs were incubated with AICAR dissolved in $0.1 \%$ DMSO in the presence of melatonin for $6 \mathrm{~h}$ (Supplementary Figure 2Aa) and (2) cells were co-incubated with AICAR, TG, and melatonin to induce ER stress in advance (Supplementary Figure $2 \mathrm{Ab}$ ) and then washed with PBS and cultured with $400 \mu \mathrm{M} \mathrm{H}_{2} \mathrm{O}_{2}$ for another $24 \mathrm{~h}$. The results presented in Supplementary Figure $2 \mathrm{~B}$ show that treatment with these two small-molecule agents at concentrations less than $500 \mu \mathrm{M}$ for $24 \mathrm{~h}$ marginally inhibited the viability of BMSCs. Additionally, at these concentrations, the expression levels of $\mathrm{p}$-AMPK and p-PERK protein were significantly promoted (Supplementary Figure 2C). We found that pretreatment with AICAR significantly promoted the protective effects of melatonin on cell viability (Fig. 7a), ROS production (Fig. 7b and Supplementary Figure 3A), mitochondrial superoxide levels (Fig. 7c and Supplementary Figure 3B), and the MMP (Fig. $7 \mathrm{~d}$ and Supplementary Figure 3C) in BMSCs exposed to $\mathrm{H}_{2} \mathrm{O}_{2}$ induced injury. Moreover, cell apoptosis was evaluated by C-Casp-3 immunofluorescence followed by TUNEL staining, as described previously. Pretreatment with AICAR and melatonin further decreased the green (TUNEL ${ }^{+}$cells) and red (C-Casp-3-labeled cells) fluorescence intensities, which indicated that the combination of these agents had a stronger antiapoptotic effect than did either agent alone (Fig. 7e-g). Additionally, AICAR treatment markedly enhanced the regulatory effect of melatonin on proteins of interest, including p-AMPK, ER stress markers (p-PERK, Bip, p-eIF2 $\alpha$, and DDIT3), apoptosis regulatory proteins (C-Casp-3, $\mathrm{Bcl} 2$, and $\mathrm{Bax}$ ), and mitochondrial oxidative stress-related proteins (Cyto-C and MnSOD) (Fig. 7h, i). Conversely, compared with AICAR and melatonin treatment, the addition of TG reduced BMSC viability (Fig. 7a), ROS production (Fig. 7b and Supplementary Figure 3A), mitochondrial superoxide levels (Fig. 7c and Supplementary Figure 3B), and the MMP (Fig. 7d and Supplementary Figure 3C) during $\mathrm{H}_{2} \mathrm{O}_{2}$-induced injury. As expected, the regulatory effects of AICAR and melatonin treatment on the apoptotic index and the abovementioned proteins were abolished after TG administration (Fig. 7e-i).

\section{Regulatory effects of inactivated AMPK and ER stress on melatonin-mediated cellular protection against $\mathrm{H}_{2} \mathrm{O}_{2}-$ induced damage}

To further analyze the relationship between AMPK and ER stress in the melatonin-mediated antiapoptotic effects during $\mathrm{H}_{2} \mathrm{O}_{2}$-induced injury, the negative regulatory reagents $\mathrm{CpC}$ and 4-PBA were used to downregulate their respective targets. As with agonist 


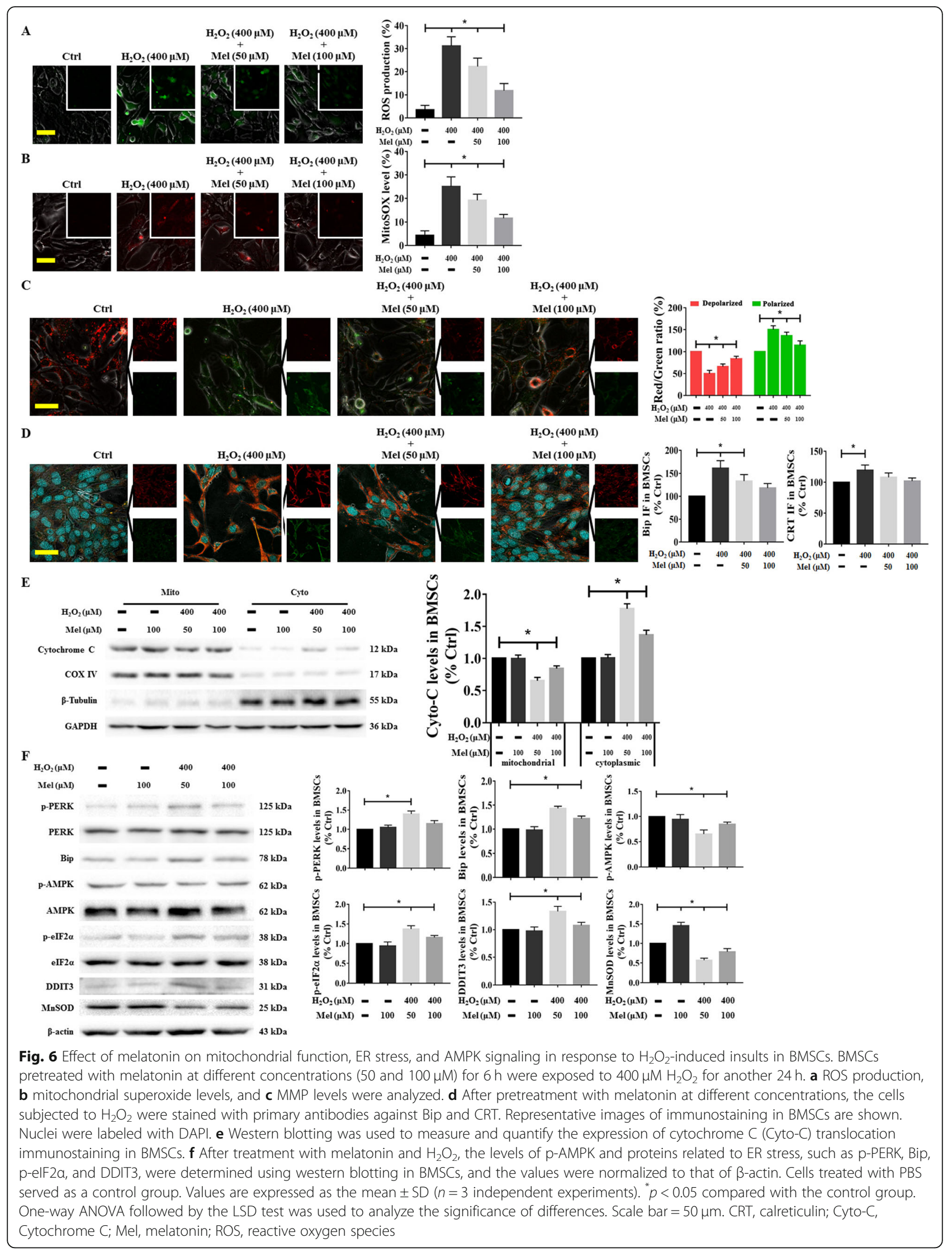




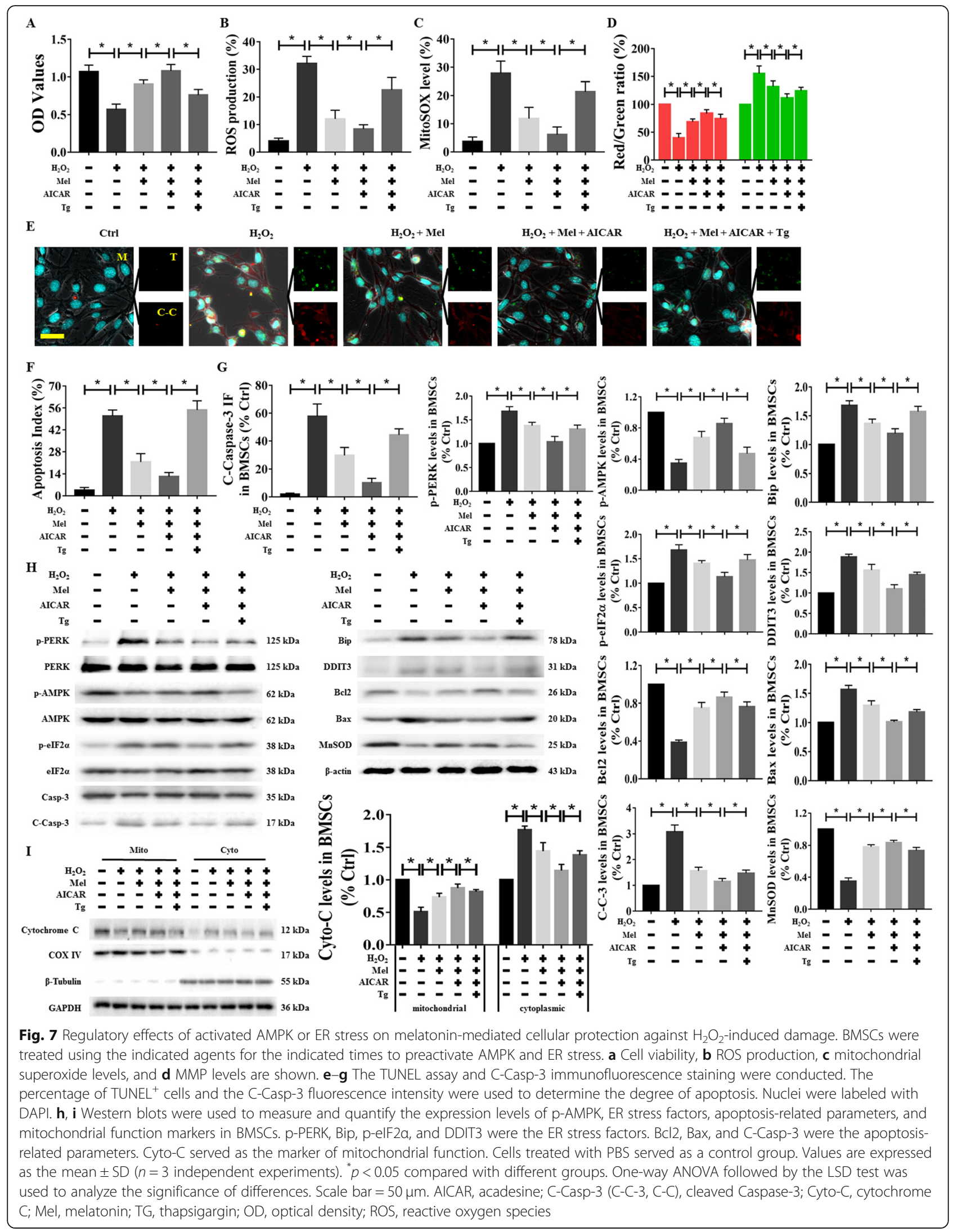


application, safe concentrations were first identified and then applied to cell cultures for the indicated periods. The CCK-8 results demonstrated that $\mathrm{CpC}$ and 4-PBA started to inhibit cell viability at concentrations of $6 \mu \mathrm{M}$ and $200 \mu \mathrm{M}$, respectively (Supplementary Figure 2D), and the expression of their target proteins was obviously inhibited at these concentrations (Supplementary Figure 2E). Therefore, in addition to being treated with $\mathrm{CpC}$ $(4 \mu \mathrm{M})$ and melatonin $(100 \mu \mathrm{M})$, the cells were coincubated with $\mathrm{CpC}(4 \mu \mathrm{M})$, 4-PBA $(100 \mu \mathrm{M})$, and melatonin $(100 \mu \mathrm{M})$ for $6 \mathrm{~h}$ and then cultured with $400 \mu \mathrm{M} \mathrm{H} \mathrm{H}_{2} \mathrm{O}_{2}$ for another $24 \mathrm{~h}$ (Supplementary Figure 2Ac and $\mathrm{Ad}$ ). Melatonin treatment obviously increased cellular activity and the MMP and decreased the ROS production, MitoSOX fluorescence, and cellular apoptosis, and these effects were reversed by $\mathrm{CpC}$ pretreatment (Fig. 8a-g and Supplementary Figure 4A-4C). Moreover, after $\mathrm{CpC}$ treatment, the melatonin-mediated downregulation of proteins (p-PERK, Bip, p-eIF2 $\alpha$, DDIT3, C-Casp-3, and $\mathrm{Bcl} 2$ ), the translocation of Cyto- $\mathrm{C}$, and the upregulation of certain proteins (Bax and $\mathrm{MnSOD}$ ) during $\mathrm{H}_{2} \mathrm{O}_{2}$-induced injury were reduced (Fig. $8 \mathrm{~h}$, i). However, treatment of the BMSCs with 4-PBA partially normalized cell functions and alleviated oxidative stress levels and apoptosis (Fig. 8a-i and Supplementary Figure 4A-4C). Additionally, AMPK siRNA and DDIT3 siRNA reagents did not affect cell activity despite effectively reducing the levels of their respective target proteins (Supplementary Figure 2F). After pretreatment with AMPK siRNA, the melatonin-mediated upregulation of cytoactivity during $\mathrm{H}_{2} \mathrm{O}_{2}$-induced injury was reduced, while the administration of DDIT3 siRNA promoted the cytoprotective effect of melatonin in BMSCs (Supplementary Figure 2Ae and G). These results provide further evidence that melatonin exerts cell-protective effects through activation of AMPK and inhibition of the ER stress pathway, as AMPK-induced increases in the protective effect of melatonin contribute to a partial reduction of ER stress in BMSCs.

\section{Discussion}

The molecular pathways responsible for the beneficial effects of BMSC transplantation as a cell-based strategy are still not fully understood, although stem cells have the potential to be used for clinical applications. Understanding the regulatory mechanisms by which BMSCs promote colonization, especially in the oxidative microenvironment after injury, will greatly contribute to improving their clinical efficacy. In this study, we first established a canonical oxidative stress injury model with $\mathrm{H}_{2} \mathrm{O}_{2}$, which is a classical signaling molecule that is commonly used to establish models of exogenous oxidative stress because of its unique biochemical properties, such as a relatively long half-life and solubility in both lipids and aqueous media [35, 36]. Then, we developed a novel strategy to preserve the biological function of BMSCs based on the specific small bioactive substance melatonin. Here, we report that treatment with $\mathrm{H}_{2} \mathrm{O}_{2}$ inhibited cell viability and induced BMSC apoptosis. From a molecular perspective, $\mathrm{H}_{2} \mathrm{O}_{2}$ insult promoted the accumulation of ROS, disrupted mitochondrial energy metabolism, promoted oxidative stress, suppressed cytoprotective signals, and initiated ER stress in BMSCs. Importantly, $\mathrm{H}_{2} \mathrm{O}_{2}$-mediated cell apoptosis, mitochondrial injury, and ER stress were prevented by melatonin administration through a mechanism involving ROS inhibition and AMPK activation. To our knowledge, this is the first investigation to demonstrate that the relationship between AMPK-modified mitochondrial injury and ER stress is sufficient to mediate $\mathrm{H}_{2} \mathrm{O}_{2}$-induced oxidative stress injury and that melatonin is an effective agent for promoting BMSC survival.

MSCs, which are important candidates for cell-based strategies, play an important role in promoting tissue repair and regeneration in multisystem diseases because they possess immunosuppressive properties and the capacity for unlimited self-amplification and terminal differentiation [37-39]. In terms of cell characteristics, MSCs isolated from the bone marrow, adipose tissue, or umbilical cord are spindle-shaped, undergo cell growth, and express the surface molecules CD73, CD105, CD90, and CD29 (>90\%) but not CD45, CD34, CD14, or CD79 [40]. However, increasing evidence suggests that the survival of donated stem cells is poor and that stem cell engraftment in the damaged organ fails after delivery, which unavoidably results from a complicated environment involving risk factors that may lead to MSC death, including oxidative stress, and ultimately hinders the clinical application of cytotherapy [41-43]. Therefore, a complete exploration of the underlying mechanisms and drugs with antioxidative effects that enhance MSC migration and survival in the injury-associated microenvironment is crucial for improving both MSC repair capacity and therapeutic applications of MSCs.

Mitochondria, which are known as the principal sources of ROS, are the main organelles involved in metabolism and respiration in eukaryotic cells and are inseparably linked to oxidative stress after disruption of mitochondrial homeostasis [32]. Maintaining the normal function of mitochondria is essential for ensuring the efficient scavenging of ROS and protecting cells from mitochondrion-mediated cell death [44]. Mitochondrial dysfunction, loss of the inner MMP, and increased mitochondrial membrane permeability represent cardinal biochemical markers of apoptosis [45]. Apoptosis induction through a mitochondria-dependent pathway typically involves many regulatory molecules, such as $\mathrm{Bcl} 2$, Bax, Cyto-C, and Caspases [46]. In our study, after 


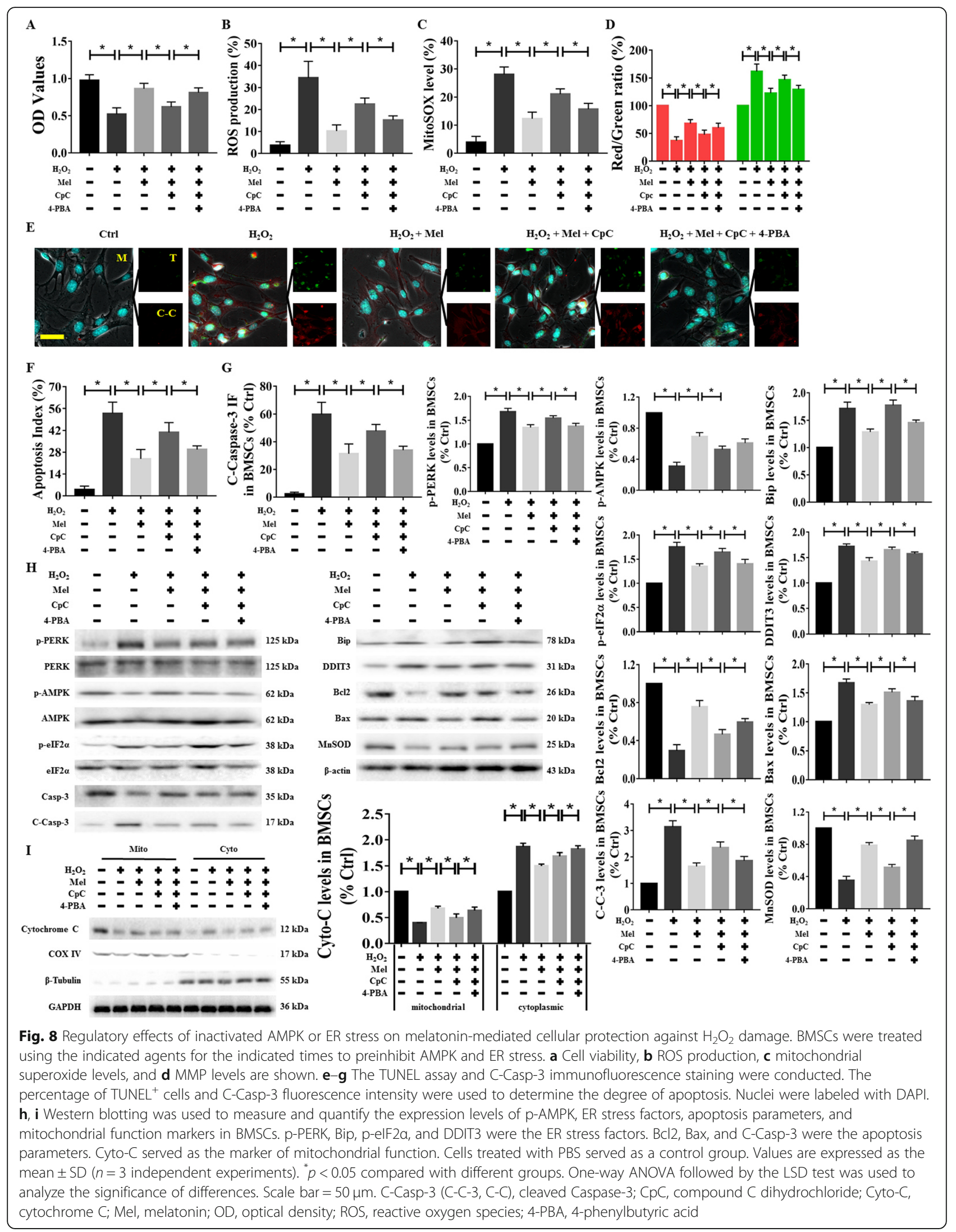


treatment with $\mathrm{H}_{2} \mathrm{O}_{2}$ (a nonradical derivative of $\mathrm{O}_{2}$ ), ROS production in the cytoplasm and superoxides in mitochondria were increased, as evidenced by DCFH-DA and MitoSOX $^{\mathrm{Tw}}$ Red staining, respectively; this observation indicated that excessive oxidative stress occurred in the cells; combined with reduced cellular activity and an increase in the number of TUNEL ${ }^{+}$cells, these results indicated that the mitochondrial apoptotic pathway was activated in BMSCs. Moreover, intracellular oxidative stress depolarizes the MMP, further promoting the recruitment of a proapoptotic protein (Bax) to the mitochondrial membrane, increasing the mitochondrial membrane permeability and thus facilitating the release of Cyto-C [47]. Conversely, the antiapoptotic protein Bcl2 protects mitochondria by inhibiting Bax activation, thus ensuring outer mitochondrial membrane integrity and inhibiting the release of Cyto-C. However, once released, Cyto- $\mathrm{C}$ cooperatively activates Caspase- 3 and other factors to exacerbate apoptotic cell death $[46,48]$; this phenomenon was confirmed by our western blot results.

The ER is an interconnected and continuous network formed by the nuclear envelope and is essentially associated with other organelles in all eukaryotic cells [49]. Structurally, the ER interacts with mitochondria at contact sites called mitochondria-associated membranes (MAMs), which facilitate interorganelle communication [50]. Functionally, the ER is critical for protein synthesis and transport to the extracellular space, mitochondrial function, the maintenance of $\mathrm{Ca}^{2+}$ homeostasis, and apoptosis [51]. Thus, the ER interior mimics the relatively oxidized extracellular environment, which causes the ER to be sensitive to perturbations inside or outside the cell, including the oxidative stress response [52]. In this sense, alterations in the oxidation state of the ER lumen can lead to an accumulation of misfolded polypeptides, causing further ER stress [53]. The oxidative folding machinery is then hyperactivated to refold improperly folded proteins; this process is accompanied by the production of ROS and ultimately disrupts ER function for an indefinite period [53]. Therefore, ROS that are mainly generated in mitochondria can perturb ER function; in contrast, under stress conditions, the ER assists with ROS production in mitochondria, leading to a vicious cycle that produces a general state of spiraling oxidative stress. It has been demonstrated that during ER stress, ROS upregulate the levels of protein chaperones (such as Bip and CRT), which play a vital role in the PERK pathway, the signaling pathway that is most closely tied to oxidative stress [52, 54]. Our results demonstrated that $\mathrm{H}_{2} \mathrm{O}_{2}$ increased PERK phosphorylation and the expression of Bip, CRT, p-eIF2a, and DDIT3, which make up the PERK pathway and contribute to signal transmission to regulate cell fate. Moreover, after oxidative stress injury, ER stress in BMSCs is associated with mitochondrial dysfunction and cell death, which may explain the mechanisms underlying ERmitochondria-induced apoptosis.

ROS produced as part of such a response to perturbations, which act primarily to restore homeostasis, can be considered a part of cellular stress signaling pathways that can mediate AMPK activation by decreasing ATP production [52, 55]. In our study, $\mathrm{H}_{2} \mathrm{O}_{2}$ injury induced AMPK dephosphorylation, possibly because oxidative stress impaired energy metabolism to induce the apoptosis of BMSCs [15]. Additionally, AMPK is an evolutionarily conserved energy sensor that has diverse cytoprotective effects $[14,15]$. A number of compounds, including melatonin, have been found to activate and thus biologically regulate AMPK [56].

Melatonin is a hormone derived from tryptophan metabolism. It is mainly synthesized and secreted by the pineal gland and has also been verified to be present at high levels in the bone marrow [19]. Our experiments showed that melatonin promoted the biological behaviors of BMSCs and their ability to maintain self-renewal and differentiation capacities after long-term passage [41], demonstrating that melatonin is a beneficial treatment for cell transplantation. Importantly, our previous findings indicated that melatonin contributes to the amelioration of high-flow shear stress-induced BMSC injury by activating melatonin receptors and AMPK/ACC signaling [4]; however, the specific regulatory mechanisms and effector pathways have not been fully revealed. Considering that melatonin and its metabolites are known to scavenge a variety of free radicals and are able to act both on every cell and within every subcellular compartment $[57,58]$, we focused on the regulatory effect of melatonin on mitochondria and the ER during $\mathrm{H}_{2} \mathrm{O}_{2}$-induced BMSC injury. Our data showed that melatonin prevented cell death induced by $\mathrm{H}_{2} \mathrm{O}_{2}$ damage, decreased the apoptotic index, and improved cell proliferation. In addition, pretreatment with melatonin relieved oxidative stress and restored mitochondrial function, as evidenced by a decrease in ROS production, an increase in the MMP, inhibition of Cyto-C translocation, and upregulation of MnSOD (an antioxidant enzyme essential for cell survival through the catalysis of the spontaneous dismutation of superoxide radicals [59]). Moreover, melatonin activated AMPK and reduced the expression of proteins related to ER stress, such as p-PERK, Bip, p-eIF2 $\alpha$, and DDIT3. As a result, melatonin exerted an antiapoptotic effect by upregulating Bax and downregulating Bcl2 and Caspase-3 activation.

To explore the relationship between ROS and apoptosis in the protective effect of melatonin against $\mathrm{H}_{2} \mathrm{O}_{2}$ injury, NAC $(1 \mu \mathrm{M})$ was used in our study. After exposure to NAC, the cytoprotective effects of melatonin on BMSC survival and intracellular ROS were enhanced. 
Moreover, Apaf-1 plays an essential role in the mitochondrial apoptotic pathway, which is stimulated by Cyto-C to activate Caspase-3 [60]. Melatonin could inhibit the expression of Apaf- 1 at the gene and protein levels after $\mathrm{H}_{2} \mathrm{O}_{2}$ insult, and NAC enhanced the above effects as well as the regulation of other apoptotic proteins (Bcl2, Bax, and Caspase-3). These results indicate that the protective effects of melatonin on cell apoptosis occurred in a mitochondria-dependent manner due to the inhibition of ROS production.

It is worth pointing out that after cotreatment with AICAR, the most commonly used AMPK activator, the protective effects of melatonin on cell proliferation, apoptosis inhibition, and mitochondrial homeostasis were significantly strengthened. In addition, ER stress was further attenuated. Conversely, disrupting the ER with TG in advance abrogated the combined effects of AICAR and melatonin. To further evaluate the relationship between AMPK and ER stress in the process of melatonin-mediated antiapoptotic effects during $\mathrm{H}_{2} \mathrm{O}_{2}$ induced injury, the negative regulatory reagents $\mathrm{CpC}$ and 4-PBA (AMPK siRNA and DDIT3 siRNA) were used to downregulate their respective targets. As expected, CpC (AMPK siRNA) markedly blocked the regulatory effects of melatonin on the functions of AMPK, $\mathrm{ER}$, and mitochondria during oxidative stress-induced apoptosis in BMSCs, while 4-PBA (DDIT3 siNA) subsequently and partially restored these effects. This is the first study to comprehensively report the multiple impacts of melatonin on AMPK, the ER, and mitochondria.

\section{Conclusions}

Here, we demonstrate the functional link between AMPK and ER stress in BMSCs and suggest that the activation of AMPK or inhibition of ER stress is a potential target for maintaining mitochondrial homeostasis and promoting cell survival. Our work revealed that melatonin is a good candidate for such effects; it restores AMPK enzyme activity and subsequently suppresses the ER stress-associated mitochondrial oxidative response, thereby inhibiting BMSC apoptosis (Fig. 9). We conclude that melatonin is a physiologically and pharmacologically relevant molecule with remarkable potential for cellbased transplantation.

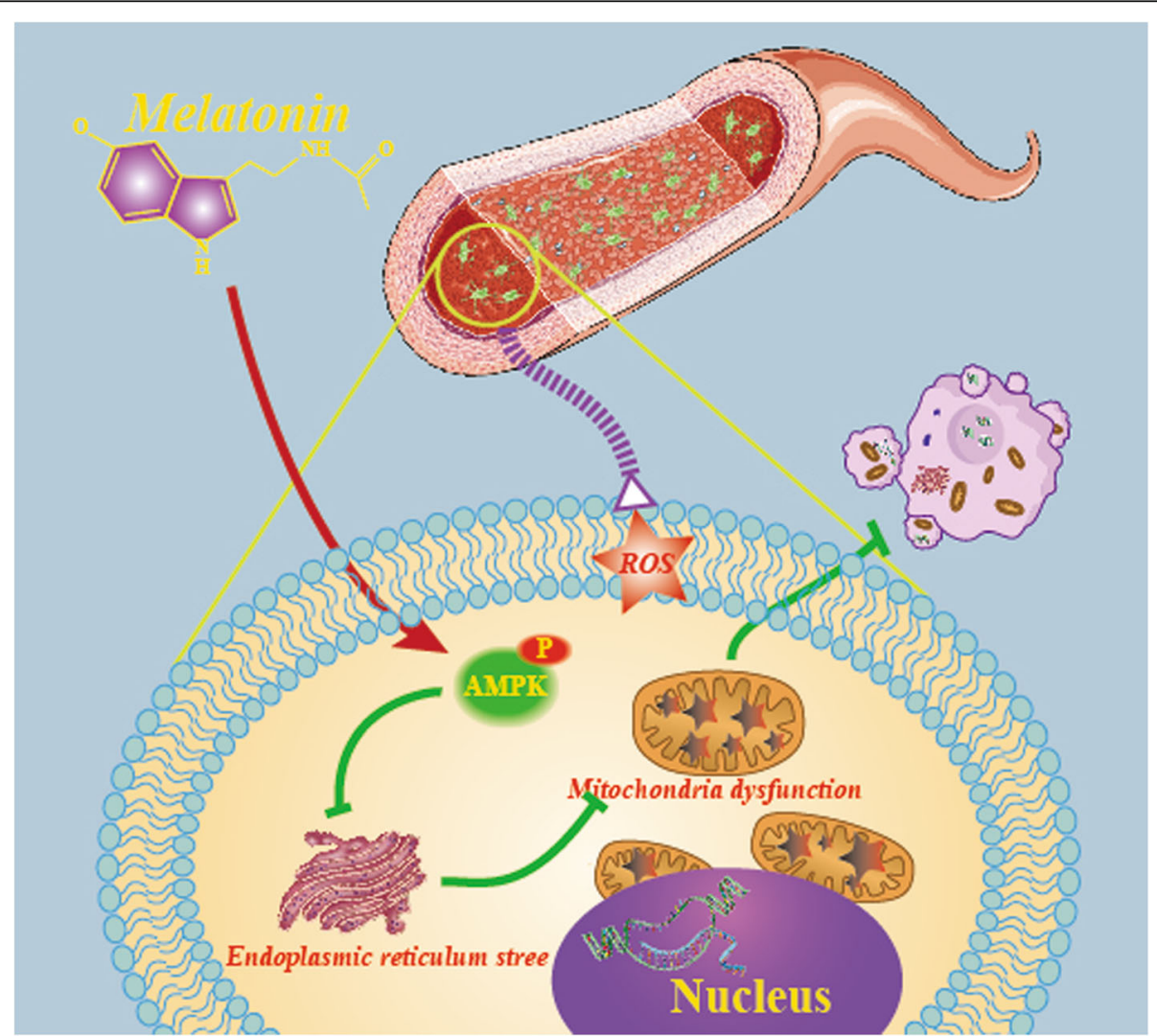

Fig. 9 Schematic diagram depicting the likely cellular events through which melatonin recovers mitochondrial homeostasis via the AMPK/ER stress pathway under $\mathrm{H}_{2} \mathrm{O}_{2}$ exposure, resulting in BMSC survival. ER stress-induced mitochondrial dysfunction plays an important role in BMSC oxidative stress injury. Melatonin treatment reduces BMSC damage and improves cell functions after $\mathrm{H}_{2} \mathrm{O}_{2}$ insult. Such cytoprotective effects seem to be largely due to the attenuation of ROS overproduction and the maintenance of mitochondrial homeostasis, which involves AMPK/ER stress signaling pathways, subsequently leading to the inhibition of cell apoptosis 


\section{Supplementary information}

Supplementary information accompanies this paper at https://doi.org/10. 1186/s13287-020-01948-5.

Additional file 1: Supplementary Figure 1: Inhibition of cellular oxidative stress on melatonin-mediated cellular protection against $\mathrm{H}_{2} \mathrm{O}_{2}$ damage. BMSCs were pretreated with NAC (1 mM) in the absence or presence of melatonin $(100 \mu \mathrm{M})$ for $6 \mathrm{~h}$ and then exposed to $400 \mu \mathrm{M}$ $\mathrm{H} 2 \mathrm{O} 2$ for another $24 \mathrm{~h}$. The (A) cell viability and (B) ROS production were demonstrated. (C-E) The TUNEL assay and C-Casp-3 immunofluorescence staining were conducted. The percentage of TUNEL ${ }^{+}$cells and C-Casp-3 fluorescence intensity were indicated to determine the apoptotic degree. Nuclei are labeled with DAPI. (F) Afpa-1 mRNA expression was analyzed by RT-qPCR. (G) Western blots were used to measure and quantify the expressions of Apaf-1, BCl2, Bax, and C-Casp-3. Cells treated with PBS were served as a control group. Values are expressed as the mean \pm SD $(n=3$ independent experiments). ${ }^{*} p<0.05$ compared with the different groups. one-way ANOVA followed by Student's t-test was used to analyze significant differences. Scale bar $=50 \mu \mathrm{m}$. C-Casp-3 (C-C-3, C-C), cleaved Caspase-3; Mel, melatonin; NAC, acetylcysteine; OD, optical density; ROS, reactive oxygen species.

Additional file 2: Supplementary Figure 2: Effect of pharmacologic agonists and inhibitors on cell viability in BMSCs. BMSCs were seeded in 96-well plates for $12 \mathrm{~h}$. (A) The experimental protocols were shown about the cotreatment melatonin with $\operatorname{AICAR}(\mathrm{a}), \mathrm{TG}(\mathrm{b}), \mathrm{CpC}(\mathrm{c})$, 4-PBA (d), AMPKsi or DDIT3si (e) followed by $\mathrm{H}_{2} \mathrm{O}_{2}(400 \mu \mathrm{M})$ for another $24 \mathrm{~h}$. (B) Different concentrations of AICAR and TG were engaged to incubate for $24 \mathrm{~h}$. The OD values were then analyzed by CCK-8. (C) Western blots were used to measure and quantify the expressions of p-AMPK or p-PERK after treated with AICAR or TG in BMSCs. (D) Different concentrations of $\mathrm{CPC}$ and 4-PBA were engaged to incubate for $24 \mathrm{~h}$. The OD values were then analyzed by CCK-8. (E) Western blots were used to measure and quantify the expressions of p-AMPK or p-PERK after treated with $\mathrm{CpC}$ or 4-PBA in BMSCs. (F) BMSCs were seeded in six-well plates and transfected with AMPK siRNA (or DDIT3 siRNA). Western blots were used to measure and quantify the expressions of AMPK or DDIT3. (G) Then the cells were incubated with melatonin for $6 \mathrm{~h}$ followed by $\mathrm{H}_{2} \mathrm{O}_{2}$ for another $24 \mathrm{~h}$. The OD values were analyzed by CCK-8. Cells treated with PBS were served as a control group. Values are expressed as the mean \pm SD ( $n=3$ independent experiments). ${ }^{*} p<0.05$ compared with the different groups. one-way ANOVA followed by Student's t-test was used to analyze significant differences. AICAR, acadesine; AMPKsi, AMPK siRNA; DDIT3si, DDIT3 siRNA; Ctrlsi, control siRNA; CpC, compound C dihydrochloride; Mel, melatonin; OD, optical density; TG, thapsigargin; 4-PBA, 4-phenylbutyric acid.

Additional file 3: Supplementary Figure 3: Regulatory effects of activated AMPK or ER stress on melatonin-mediated homeostasis about ROS and mitochondrial function. BMSCs were treated as indicated agents and time to pre-activate AMPK and ER stress. The fluorescent photograph on (A) intracellular ROS, (B) mitochondrial superoxide, and (C) mitochondrial membrane potential were demonstrated $(n=3$ independent experiments). Scale bar $=50 \mu \mathrm{m}$. AICAR, acadesine; Mel, melatonin; TG,

thapsigargin

Additional file 4: Supplementary Figure 4: Regulatory effects of inactivated AMPK or ER stress on melatonin-mediated homeostasis about ROS and mitochondrial function. BMSCs were treated as indicated agents and time to pre-inhibit AMPK and ER stress. The fluorescent photograph on (A) intracellular ROS, (B) mitochondrial superoxide, and (C) mitochondrial membrane potential were demonstrated $(n=3$ independent experiments). Scale bar $=50 \mu \mathrm{m}$. CpC, compound $\mathrm{C}$ dihydrochloride; Mel, melatonin; 4-PBA, 4-phenylbutyric acid.

\section{Abbreviations}

4-PBA: 4-Phenylbutyric acid; ACC: Acetyl-Co A carboxylase; AICAR: Acadesine; AMP: Adenosine monophosphate; AMPK: AMP-activated protein kinase; ATF6: Activating transcription factor 6; ATP: Adenosine triphosphate; BCA: Bicinchoninic acid; BM: Basal medium; BMSCs: Bone marrow mesenchymal stem cells; BSA: Bovine serum albumin; C-Casp-3 (C-C-3, CC): Cleaved Caspase-3; CCK-8: Cell counting kit-8; COX IV: Cytochrome C oxidase IV; CpC: Compound C dihydrochloride; CRT: Calreticulin; Cyto-
C: Cytochrome C; DAPI: 4',6-Diamidino-2-phenylindole; DDIT3: DNA damageinducible transcript 3; EDTA: Ethylenediaminetetraacetic acid; FBS: Fetal bovine serum; $\mathrm{H}_{2} \mathrm{O}_{2}$ : Hydrogen peroxide; HBSS: Hanks balanced salt solution; IRE1: Inositol-requiring protein 1; JC-1: 5,5',6,6'-Tetraethylbenzimidazolylcarbocyanine iodide; LDH: Lactate dehydrogenase; MAMs: Mitochondria-associated membranes; Mel: Melatonin; MMP: Mitochondrial membrane potential; NAC: N-acetyl-L-cysteine; OD: Optical density; P4: Passage 4; PERK: RNA-dependent protein kinase (PKR)-like ER kinase; p-PERK: Phospho-PERK; PI: Propidium iodide; RIPA: Radioimmunoprecipitation assay; RNS: Reactive nitrogen species; ROS: Reactive oxygen species; RT-qPCR: Real-time quantitative PCR: SD: Standard deviation; siRNA: Small interfering RNA; UPR: Unfolded protein response; TUNEL: Terminal deoxynucleotidyl transferase dUTP nick end labeling

\section{Acknowledgements}

We sincerely appreciate the excellent assistance from Professor Jintao Hu (Department of Immunology, Fourth Military Medical University) with the flow cytometry experiments.

\section{Authors' contributions}

E.P.L. and C.X.F. designed the research and revised the manuscript; C.X.F., J.Y.F., and C.T. performed the experiments and drafted the manuscript; Z.B.Z., Y.T.F., W.X.D., and L.W.Z. collected and analyzed the data; and M.M.Z., Z.D.Y., L.L.F., and H.Z.Z. prepared the figures. The authors read and approved the manuscript

\section{Funding}

This work was supported by the National Natural Science Foundation of China (NSFC) (grant Nos. 81702731, 51907111, and 11972366) and the Key Project supported by the Medical Science and Technology Development Foundation of the Shaanxi Department of Health (grant Nos. 2018SF-159, 2018SF-176, and 2020SF-084).

\section{Availability of data and materials}

The datasets used and/or analyzed during the current study are available from the corresponding author on reasonable request.

\section{Ethics approval and consent to participate}

This study was approved by the Ethics Committee of the Fourth Military Medical University (No. 20180301).

\section{Consent for publication}

Not applicable.

\section{Competing interests}

The authors declare that they have no conflicts of interest related to the studies described.

\section{Author details}

${ }^{1}$ Department of Military Biomedical Engineering, Air Force Medical University, 169 Changle West Road, Xi'an 710032, China. ${ }^{2}$ Department of Oncology, Air Force Medical Center of PLA, 30 Fucheng Road, Beijing 100142, China. ${ }^{3}$ Department of Cardiovascular Surgery, Xijing Hospital, Air Force Medical University, 127 Changle West Road, Xi'an 710032, China. ${ }^{4}$ Department of Geriatrics, The 8th Medical Center of Chinese PLA General Hospital, 17 Heishanhu Street, Beijing 100091, China. ${ }^{5}$ Department of Thoracic Surgery, Tangdu Hospital, Air Force Medical University, 1 Xinsi Road, Xi'an 710038, China. ${ }^{6}$ Department of Cardiology, The First Affiliated Hospital of Xi'an Medical University, 277 Yanta West Road, Xi'an 710077, China.

Received: 21 May 2020 Accepted: 23 September 2020 Published online: 15 October 2020

\section{References}

1. Uccelli A, Moretta L, Pistoia V. Mesenchymal stem cells in health and disease. Nat Rev Immunol. 2008;8:726-36.

2. Mias C, Trouche E, Seguelas M-H, Calcagno F, Dignat-George F, Sabatier F, et al. Ex vivo pretreatment with melatonin improves survival, proangiogenic/mitogenic activity, and efficiency of mesenchymal stem cells injected into ischemic kidney. Stem Cells (Dayton, Ohio). 2008;26:1749-57. 
3. Kavanagh DPJ, Suresh S, Newsome PN, Frampton J, Kalia N. Pretreatment of mesenchymal stem cells manipulates their vasculoprotective potential while not altering their homing within the injured gut. Stem Cells (Dayton, Ohio). 2015:33:2785-97.

4. Yang Y, Fan C, Deng C, Zhao L, Hu W, Di S, et al. Melatonin reverses flow shear stress-induced injury in bone marrow mesenchymal stem cells via activation of AMP-activated protein kinase signaling. J Pineal Res. 2016;60: 228-41.

5. Bailey KA, Moreno E, Haj FG, Simon SI, Passerini AG. Mechanoregulation of p38 activity enhances endoplasmic reticulum stress-mediated inflammation by arterial endothelium. FASEB J. 2019;33:12888-99.

6. Dixit N, Kim M-H, Rossaint J, Yamayoshi I, Zarbock A, Simon SI. Leukocyte function antigen-1, kindlin-3, and calcium flux orchestrate neutrophil recruitment during inflammation. J Immunol (Baltimore, Md. : 1950). 2012; 189:5954-64.

7. Hetz C, Chevet E, Harding HP. Targeting the unfolded protein response in disease. Nat Rev Drug Discov. 2013;12:703-19.

8. Grootjans J, Kaser A, Kaufman RJ, Blumberg RS. The unfolded protein response in immunity and inflammation. Nat Rev Immunol. 2016;16:469-84.

9. Kim I, Xu W, Reed JC. Cell death and endoplasmic reticulum stress: disease relevance and therapeutic opportunities. Nat Rev Drug Discov. 2008;7:101330.

10. Chiu H-W, Xia T, Lee Y-H, Chen C-W, Tsai J-C, Wang Y-J. Cationic polystyrene nanospheres induce autophagic cell death through the induction of endoplasmic reticulum stress. Nanoscale. 2015;7:736-46.

11. Kass GE, Orrenius S. Calcium signaling and cytotoxicity. Environ Health Perspect. 1999;107(Suppl 1):25-35.

12. Oakhill JS, Steel R, Chen Z-P, Scott JW, Ling N, Tam S, et al. AMPK is a direct adenylate charge-regulated protein kinase. Science (New York, N.Y.). 2011; 332:1433-5.

13. Gowans GJ, Hawley SA, Ross FA, Hardie DG. AMP is a true physiological regulator of AMP-activated protein kinase by both allosteric activation and enhancing net phosphorylation. Cell Metab. 2013;18:556-66.

14. Kahn BB, Alquier T, Carling D, Hardie DG. AMP-activated protein kinase: ancient energy gauge provides clues to modern understanding of metabolism. Cell Metab. 2005;1:15-25

15. Yang S-H, Li W, Sumien N, Forster M, Simpkins JW, Liu R. Alternative mitochondrial electron transfer for the treatment of neurodegenerative diseases and cancers: methylene blue connects the dots. Prog Neurobiol. 2017;157:273-91.

16. Garcia D, Shaw RJ. AMPK: mechanisms of cellular energy sensing and restoration of metabolic balance. Mol Cell. 2017:66:789-800.

17. Herzig S, Shaw RJ. AMPK: guardian of metabolism and mitochondrial homeostasis. Nature reviews Mol Cell Biol 2018;19:121-135.

18. Lu J, Wang Q, Huang L, Dong H, Lin L, Lin N, et al. Palmitate causes endoplasmic reticulum stress and apoptosis in human mesenchymal stem cells: prevention by AMPK activator. Endocrinology. 2012;153:5275-84.

19. Tan DX, Manchester LC, Reiter RJ, Qi WB, Zhang M, Weintraub ST, et al Identification of highly elevated levels of melatonin in bone marrow: its origin and significance. Biochim Biophys Acta. 1999;1472:206-14.

20. Jan JE, Reiter RJ, Wasdell MB, Bax M. The role of the thalamus in sleep, pineal melatonin production, and circadian rhythm sleep disorders. J Pineal Res. 2009:46:1-7.

21. Lee JH, Yoon YM, Han Y-S, Jung SK, Lee SH. Melatonin protects mesenchymal stem cells from autophagy-mediated death under ischaemic ER-stress conditions by increasing prion protein expression. Cell Prolif. 2019; 52:e12545.

22. Liu X, Gong Y, Xiong K, Ye Y, Xiong Y, Zhuang Z, et al. Melatonin mediates protective effects on inflammatory response induced by interleukin-1 beta in human mesenchymal stem cells. J Pineal Res. 2013;55:14-25.

23. Soleimani M, Nadri S. A protocol for isolation and culture of mesenchymal stem cells from mouse bone marrow. Nat Protoc. 2009:4:102-6.

24. Zhang Z, Zhu L, Feng P, Tan Y, Zhang B, Gao E, et al. C1 q/tumor necrosis factorrelated protein-3-engineered mesenchymal stromal cells attenuate cardiac impairment in mice with myocardial infarction. Cell Death Dis. 2019;10:530.

25. Wu B, Feng J-Y, Yu L-M, Wang $Y$ - C, Chen $Y-Q$, Wei $Y$, et al. Icariin protects cardiomyocytes against ischaemia/reperfusion injury by attenuating sirtuin 1-dependent mitochondrial oxidative damage. Br J Pharmacol. 2018;175: 4137-53.

26. Han N, Zhang F, Li G, Zhang X, Lin X, Yang H, et al. Local application of IGFBP5 protein enhanced periodontal tissue regeneration via increasing the migration, cell proliferation and osteo/dentinogenic differentiation of mesenchymal stem cells in an inflammatory niche. Stem Cell Res Ther. 2017; $8: 210$

27. Liang Y, Fan C, Yan X, Lu X, Jiang H, Di S, et al. Berberine ameliorates lipopolysaccharide-induced acute lung injury via the PERK-mediated Nrf2/ HO-1 signaling axis. Phytother Res. 2019;33:130-48.

28. Zheng Z, Zhang L, Qu Y, Xiao G, Li S, Bao S, et al. Mesenchymal stem cells protect against hypoxia-ischemia brain damage by enhancing autophagy through brain derived neurotrophic factor/mammalin target of rapamycin signaling pathway. Stem Cells (Dayton, Ohio). 2018;36:1109-21.

29. Tejero J, Shiva S, Gladwin MT. Sources of vascular nitric oxide and reactive oxygen species and their regulation. Physiol Rev. 2019;99:311-79.

30. Zhong J, Tan Y, Lu J, Liu J, Xiao X, Zhu P, et al. Therapeutic contribution of melatonin to the treatment of septic cardiomyopathy: a novel mechanism linking Ripk3-modified mitochondrial performance and endoplasmic reticulum function. Redox Biol. 2019;26:101287

31. Fang J, Zhao X, Li S, Xing X, Wang H, Lazarovici P, et al. Protective mechanism of artemisinin on rat bone marrow-derived mesenchymal stem cells against apoptosis induced by hydrogen peroxide via activation of cRaf-Erk1/2-p90-CREB pathway. Stem Cell Res Ther. 2019;10:312.

32. Vringer E, Tait SWG. Mitochondria and inflammation: cell death heats up. Front Cell Dev Biol. 2019;7:100

33. Ye Z-W, Zhang J, Ancrum T, Manevich Y, Townsend DM, Tew KD. Glutathione S-transferase P-mediated protein S-glutathionylation of resident endoplasmic reticulum proteins influences sensitivity to drug-induced unfolded protein response. Antioxid Redox Signal. 2017;26:247-61.

34. Zafarullah M, Li WQ, Sylvester J, Ahmad M. Molecular mechanisms of Nacetylcysteine actions. Cell Mol Life Sci. 2003;60(1):6-20.

35. Dröge W. Free radicals in the physiological control of cell function. Physiol Rev. 2002:82:47-95.

36. Ryter SW, Kim HP, Hoetzel A, Park JW, Nakahira K, Wang X, et al. Mechanisms of cell death in oxidative stress. Antioxid Redox Signal. 2007;9: 49-89.

37. Galipeau J, Sensébé L. Mesenchymal stromal cells: clinical challenges and therapeutic opportunities. Cell Stem Cell. 2018;22:824-33.

38. Pittenger MF, Mackay AM, Beck SC, Jaiswal RK, Douglas R, Mosca JD, et al. Multilineage potential of adult human mesenchymal stem cells. Science (New York, N.Y.). 1999;284:143-7.

39. Prockop DJ. Marrow stromal cells as stem cells for nonhematopoietic tissues. Science (New York, N.Y.). 1997;276:71-4.

40. Yao G, Qi J, Liang J, Shi B, Chen W, Li W, et al. Mesenchymal stem cell transplantation alleviates experimental Sjögren's syndrome through IFN- $\beta$ / IL-27 signaling axis. Theranostics. 2019;9:8253-65.

41. Shuai Y, Liao L, Su X, Yu Y, Shao B, Jing H, et al. Melatonin treatment improves mesenchymal stem cells therapy by preserving stemness during long-term in vitro expansion. Theranostics. 2016;6:1899-917.

42. Boopathy AV, Pendergrass KD, Che PL, Yoon Y-S, Davis ME. Oxidative stressinduced Notch1 signaling promotes cardiogenic gene expression in mesenchymal stem cells. Stem Cell Res Ther. 2013:4:43.

43. Yan W, Guo Y, Tao L, Lau WB, Gan L, Yan Z, et al. C1 q/tumor necrosis factorrelated protein-9 regulates the fate of implanted mesenchymal stem cells and mobilizes their protective effects against ischemic heart injury via multiple novel signaling pathways. Circulation. 2017;136:2162-77.

44. Hou J, Han ZP, Jing YY, Yang X, Zhang SS, Sun K, et al. Autophagy prevents irradiation injury and maintains stemness through decreasing ROS generation in mesenchymal stem cells. Cell Death Dis. 2013;4:e844.

45. Kroemer G, Dallaporta B, Resche-Rigon M. The mitochondrial death/life regulator in apoptosis and necrosis. Annu Rev Physiol. 1998;60:619-42.

46. Negrette-Guzmán M, Huerta-Yepez S, Tapia E, Pedraza-Chaverri J. Modulation of mitochondrial functions by the indirect antioxidant sulforaphane: a seemingly contradictory dual role and an integrative hypothesis. Free Radic Biol Med. 2013;65:1078-89.

47. Goping IS, Gross A, Lavoie JN, Nguyen M, Jemmerson R, Roth K, et al. Regulated targeting of BAX to mitochondria. J Cell Biol. 1998;143:207-15.

48. Kornicka K, Houston J, Marycz K. Dysfunction of mesenchymal stem cells isolated from metabolic syndrome and type 2 diabetic patients as result of oxidative stress and autophagy may limit their potential therapeutic use. Stem Cell Rev Rep. 2018;14:337-45.

49. Silva-Palacios A, Zazueta C, Pedraza-Chaverri J. ER membranes associated with mitochondria: possible therapeutic targets in heart-associated diseases. Pharmacol Res. 2020;156:104758. 
50. Copeland DE, Dalton AJ. An association between mitochondria and the endoplasmic reticulum in cells of the pseudobranch gland of a teleost. J Biophys Biochem Cytol. 1959;5:393-6.

51. Eisner V, Picard M, Hajnóczky G. Mitochondrial dynamics in adaptive and maladaptive cellular stress responses. Nat Cell Biol. 2018;20:755-65.

52. Ochoa CD, Wu RF, Terada LS. ROS signaling and ER stress in cardiovascular disease. Mol Asp Med. 2018;63:18-29.

53. Murphy MP. Mitochondrial dysfunction indirectly elevates ROS production by the endoplasmic reticulum. Cell Metab. 2013;18:145-6.

54. Balsa E, Soustek MS, Thomas A, Cogliati S, García-Poyatos C, Martín-García E, et al. ER and nutrient stress promote assembly of respiratory chain supercomplexes through the PERK-elF2a Axis. Mol Cell. 2019;74(5):877-90.

55. Brehm A, Krssak M, Schmid Al, Nowotny P, Waldhäusl W, Roden M. Increased lipid availability impairs insulin-stimulated ATP synthesis in human skeletal muscle. Diabetes. 2006:55:136-40.

56. Phillipson OT. Alpha-synuclein, epigenetics, mitochondria, metabolism, calcium traffic, \& circadian dysfunction in Parkinson's disease. An integrated strategy for management. Ageing Res Rev. 2017;40:149-67.

57. Pandi-Perumal SR, Srinivasan V, Maestroni GJM, Cardinali DP, Poeggeler B, Hardeland R. Melatonin: nature's most versatile biological signal? FEBS J. 2006;273:2813-38.

58. Hardeland R, Pandi-Perumal SR, Cardinali DP. Melatonin. Int J Biochem Cell Biol. 2006;38:313-6.

59. Chen W, Chen X, Chen AC, Shi Q, Pan G, Pei M, et al. Melatonin restores the osteoporosis-impaired osteogenic potential of bone marrow mesenchymal stem cells by preserving SIRT1-mediated intracellular antioxidant properties. Free Radic Biol Med. 2020;146:92-106.

60. Yuan J, Yankner BA. Apoptosis in the nervous system. Nature. 2000;407:802-9.

\section{Publisher's Note}

Springer Nature remains neutral with regard to jurisdictional claims in published maps and institutional affiliations.

Ready to submit your research? Choose BMC and benefit from:

- fast, convenient online submission

- thorough peer review by experienced researchers in your field

- rapid publication on acceptance

- support for research data, including large and complex data types

- gold Open Access which fosters wider collaboration and increased citations

- maximum visibility for your research: over $100 \mathrm{M}$ website views per year

At $\mathrm{BMC}$, research is always in progress.

Learn more biomedcentral.com/submissions 